\title{
Genome-wide identification and expression analysis of the bZIP transcription factors, and functional analysis in response to drought and cold stresses in pear (Pyrus breschneideri)
}

\author{
Ming $\mathrm{Ma}^{\dagger}$, Qiming Chen ${ }^{\dagger}$, Huizhen Dong, Shaoling Zhang and Xiaosan Huang ${ }^{*}$
}

\begin{abstract}
Background: Transcription factors (TFs) are involved in many important biological processes, including cell stretching, histological differentiation, metabolic activity, seed storage, gene regulation, and response to abiotic and biotic stresses. Little is known about the functions, evolutionary history, and expression patterns of basic region-leucine zipper TF family genes in pear, despite the release of the genome of Chinese white pears ("Dangshansuli").

Results: Overall, 92 bZIP genes were identified in the pear genome (Pyrus breschneideri). Of these, 83 were randomly distributed on all 17 chromosomes except chromosome 4, and the other 9 genes were located on loose scaffolding. The genes were divided into 14 subgroups. Whole-genome duplications, dispersed duplication, and purifying selection for whole-genome duplications are the main reasons for the expansion of the PbrbZIP gene family. The analysis of functional annotation enrichment indicated that most of the functions of PbrbZIP genes were enriched in Gene Ontology and Kyoto Encyclopedia of Genes and Genomes pathways involved in the abiotic stress response. Next, expression analysis and virus-induced gene silencing results indicated that PbrbZIP genes might play critical roles in response to drought and cold stresses, especially for the genes from subgroups A, C, G, I, and $S$.

Conclusions: Ninety-two PbrbZIP genes were identified from the pear genome and classified into 14 subgroups. PbrbZIP genes were mainly expanded from whole-genome duplications and dispersed duplications and retained by purifying selection. PbrbZIP genes were induced by cold and drought stresses and played important roles in drought and cold tolerance. These results provided useful information for further increasing the tolerance of pears to stresses and a foundation to study the cold and drought tolerance mechanism of PbrbZIP genes.
\end{abstract}

Keywords: bZIP transcription factor family, Chinese white pears, Drought and cold stress tolerance, Evolutionary pattern, Gene expression

*Correspondence: huangxs@njau.edu.cn

${ }^{+}$Ming Ma and Qiming Chen contributed equally to this work.

State Key Laboratory of Crop Genetics and Germplasm Enhancement, Centre of Pear Engineering Technology Research, Nanjing Agricultural University, Nanjing, China

\begin{abstract}
Background
Transcription factors (TFs) play essential regulatory roles in many crucial biological processes in plants. Knowing the functional properties of TFs by understanding the biological processes in which they are involved is necessary. Up to now, about $64 \mathrm{TF}$ families have been reported in plants [1]. The basic family of leucine zippers (bZIP) is one of the largest and most diverse families $[1,2]$. They are characterized by a conserved bZIP domain of $40-80$
\end{abstract}


amino acids, with 2 structural features. A basic region of DNA binding (N-X7-R/K-X9) was used for sequence-specific DNA binding, and a several-heptad repeat sequence consisting of leucine or other hydrophobic amino acids (such as Ile, Val, Phe, or Met) made up the bZIP motif for dimeric specificity [2-4].

bZIP TFs are involved in several important biological processes, such as cell stretching $[5,6]$, histological differentiation [7, 8], metabolic activity [9], seed storage protein gene regulation, and embryogenesis and seed maturation [10]. bZIP TFs take part in responding to abiotic and biotic stresses, including hormone and sugar signaling $[11,12]$, photoreaction $[13,14]$, pathogen defense $[15,16]$, and abiotic stresses tolerance $[17,18]$. According to existing studies, bZIP TF plays an important role in plant response to abiotic stresses, such as drought, cold, salt, abscisic acid (ABA), and mechanical damage $[19,20]$. In soybeans, GmbZIP44, GmbZIP62, or GmbZIP78 TFs may enhance salt and cold tolerance [21]. OsbZIP62 intervenes in the signaling pathways of $\mathrm{ABA}$ and regulates positively the drought tolerance of rice by regulating the expression of genes associated with stress [22]. ZmbZIP4 TF can enhance the ability of corn to resist abiotic stresses by regulating ABA synthesis and root development [23]. In grapes, VlbZIP36 improves drought tolerance due to the transcriptional regulation of ABA/stress-related genes [24]. MdHY5 positively modulates the cold tolerance in apple calli [25].

To date, the bZIP TF families were identified or predicted across multiple plant genomes. A total of 75 bZIP genes were first found in Arabidopsis thaliana [3]. Wolfgang Drföge-Laser and co-workers classified the 78 bZIP members of $A$. thaliana into 13 subgroups [26], 89 in rice (Oryza sativa) [2], 131 in soybean (Glycine max) [21], 125 in maize (Zea mays) [27], 55 in the grapevine (Vitis vinifera) genome [28], and 112 bZIP genes in apple (Malus domestica Borkh) [29]. No studies have reported on the bZIP family in pears despite pears being an important cash crop widespread worldwide.

In fact, abiotic stresses, such as low temperature and drought, not only limit the cultivation area but also affect the growth and yield of pears. This situation needs to be addressed urgently. PbrBAM3 increases the cold tolerance of pears by increasing the antioxidant activity and soluble sugar levels [30]. PbrWRKY53 positively regulates ascorbic acid (AsA) biosynthetic activity and enhances the drought tolerance of pears by regulating AsA-mediated reactive oxygen species (ROS) scavenging [31]. Recent advances in genomics and gene technology provide many new molecular tools for improving crop resistance to biological stresses [32]. The genome sequence of Pyrus bretschneideri was released in 2013 [33], providing an opportunity for genome-level identification, analysis of protein families, and genetic improvement using candidate genes for stress resistance.

In this study, 92 PbrbZIP genes were identified from the Chinese white pear genome. Sequence and phylogenetic analyses were performed to determine the relationships among these genes. The results of the analysis of protein profiles and intron/exon structures supported the classification of the PbrbZIP family. Whole-genome duplications (WGD)/segmental and dispersed duplications probably led to the expansion of the $b Z I P$ family. In addition, RNA-seq data showed that PbrbZIP genes had different expression patterns under drought and cold stresses. The results of this study might help better understand the role of bZIP TF in the abiotic stresses response of pears and provide a foundation for identifying candidate genes involved in the cold and drought tolerance of pears.

\section{Results}

Identification of bZIP TFs in Chinese white pears

Local Hidden Markov Model (HMM) files (PF00170, PF07716, and PF07777) were used to identify the bZIP gene in the Chinese white pear genome. A total of 96 candidate PbrbZIP protein sequences were identified. The Simple Modular Architecture Research Tool (SMART) (http://smart.embl-heidelberg.de/) and the National Center for Biotechnology Information (NCBI) Batch $\mathrm{CD}$-Search tools were used to check for the presence of bZIP conserved domains, and redundant sequences were removed. A total of 92 putative $b Z I P$ genes were identified; the nomenclature and associated information are listed in Table 1 and Table S1. These PbrbZIP genes were named through PbrbZIP01 to PbrbZIP92 based on the order of the gene ID. A total of 83 PbrbZIP genes were randomly distributed on all 17 chromosomes except chromosome 4, and the other 9 genes were located on loose scaffoldings. Chromosome 15 had the most $\mathrm{Pbr}$ $b Z I P$ genes (11 genes), and chromosome 16 had only one gene. Protein molecular weights of PbrbZIP genes ranged from 14.03 to $79.84 \mathrm{KDa}$. Protein isoelectric points ranged from 5.04 to 10.51 , with 54 below 7 (Table 1). The PbrbZIP proteins might be soluble because of their positive grand average of hydropathy, which was consistent with its potential function as TF.

\section{Phylogenetic analysis and classification of the pear bZIP gene family}

An unrooted neighbor-joining phylogenetic tree was built to classify these genes, and the evolutionary relationship of the PbrbZIP gene was studied (Figs. 1, 2a, and S1). PbrbZIP genes were divided into 14 subgroups (A, B, C, D, E, F, G, H, I, J, K, M, S, and UN) based on the relationship with $A$. thaliana $b Z I P$ genes. PbrbZIPO3, 
Table 1 Characteristics of identified PbrbZIP proteins

\begin{tabular}{|c|c|c|c|c|c|c|c|c|c|}
\hline Name & ID & Chr.No & ORF & Strat & End & Extron num & $M W(K D A)$ & $\mathrm{PI}$ & GRAVY \\
\hline PbrbZIP01 & Pbro01076.1 & $\mathrm{Chr} 2$ & 702 & 12231889 & $1.2 \mathrm{E}+07$ & 1 & 26.49 & 10.41 & -0.685 \\
\hline PbrbZIP02 & Pbr002338.1 & scaffold1099.0 & 1260 & 54879 & 61696 & 12 & 43.16 & 6.62 & -0.801 \\
\hline PbrbZIP03 & Pbr002470.1 & Chr17 & 618 & 16705451 & $1.7 \mathrm{E}+07$ & 4 & 23.73 & 8.95 & -0.618 \\
\hline PbrbZIP04 & Pbr002622.1 & Chr15 & 510 & 999456 & 1002190 & 4 & 18.34 & 9.34 & -0.931 \\
\hline PbrbZIP05 & Pbr002928.1 & Chr7 & 726 & 12429663 & $1.2 \mathrm{E}+07$ & 4 & 27.24 & 9.51 & -0.907 \\
\hline PbrbZIP06 & Pbr002981.1 & Chr7 & 726 & 12802639 & $1.3 \mathrm{E}+07$ & 4 & 27.24 & 9.51 & -0.907 \\
\hline PbrbZIP07 & Pbro03516.1 & Chr3 & 1263 & 16858552 & $1.7 \mathrm{E}+07$ & 4 & 46.99 & 6.58 & -0.875 \\
\hline PbrbZIP08 & Pbro03518.1 & Chr3 & 1542 & 16831076 & $1.7 \mathrm{E}+07$ & 12 & 56.42 & 6.03 & -0.548 \\
\hline PbrbZIP09 & Pbr003750.1 & scaffold1170.0 & 726 & 35506 & 37807 & 4 & 27.18 & 9.58 & -0.876 \\
\hline PbrbZIP10 & Pbr004364.1 & Chr12 & 1461 & 2057108 & 2060551 & 6 & 53.27 & 6.21 & -0.851 \\
\hline PbrbZIP11 & Pbr005556.1 & scaffold1282.0 & 1584 & 9370 & 12132 & 4 & 57.59 & 6.79 & -0.912 \\
\hline PbrbZIP12 & Pbr005557.1 & scaffold1282.0 & 1734 & 15287 & 18296 & 4 & 62.81 & 6.63 & -0.91 \\
\hline PbrbZIP13 & Pbro05860.1 & Chr15 & 1734 & 2664599 & 2667542 & 4 & 62.88 & 6.66 & -0.901 \\
\hline PbrbZIP14 & Pbr005861.1 & Chr15 & 1584 & 2671458 & 2674216 & 4 & 57.57 & 6.79 & -0.918 \\
\hline PbrbZIP15 & Pbr005914.1 & Chr15 & 474 & 3000081 & 3001635 & 1 & 17.69 & 7.07 & -0.731 \\
\hline PbrbZIP16 & Pbro06046.1 & scaffold1301.0 & 669 & 48 & 1811 & 4 & 24.19 & 9.22 & -0.623 \\
\hline PbrbZIP17 & Pbro07163.1 & Chr14 & 639 & 14914116 & $1.5 \mathrm{E}+07$ & 1 & 22.57 & 7.84 & -0.661 \\
\hline PbrbZIP18 & Pbro07566.2 & Chr14 & 1740 & 135984 & 139566 & 6 & 62.38 & 7.59 & -0.746 \\
\hline PbrbZIP19 & Pbr007589.1 & Chr14 & 1326 & 344099 & 346885 & 4 & 48.15 & 8.28 & -0.616 \\
\hline PbrbZIP20 & Pbr008557.1 & Chr8 & 1086 & 2230491 & 2232813 & 8 & 40.94 & 6.81 & -0.528 \\
\hline PbrbZIP21 & Pbro08558.1 & Chr8 & 987 & 2197741 & 2199160 & 3 & 36.48 & 9.32 & -0.629 \\
\hline PbrbZIP22 & Pbro09074.1 & Chr10 & 1047 & 10127839 & $1 \mathrm{E}+07$ & 6 & 35.36 & 9.97 & -0.842 \\
\hline PbrbZIP23 & Pbro09262.1 & Chr15 & 1047 & 3989301 & 3992176 & 6 & 37.95 & 5.37 & -0.591 \\
\hline PbrbZIP24 & Pbro09654.1 & Chr7 & 2229 & 1362620 & 1366901 & 2 & 79.84 & 6.02 & -0.558 \\
\hline PbrbZIP25 & Pbr009693.1 & Chr7 & 510 & 1703894 & 1704403 & 1 & 18.99 & 6.23 & -0.876 \\
\hline PbrbZIP26 & Pbr010436.1 & scaffold170.2.1 & 969 & 93882 & 98146 & 3 & 35.94 & 5.94 & -0.842 \\
\hline PbrbZIP27 & Pbr010517.1 & Chr5 & 1047 & 2957830 & 2961646 & 4 & 38.24 & 6.71 & -0.936 \\
\hline PbrbZIP28 & Pbr012802.1 & Chr2 & 1278 & 5526442 & 5528863 & 6 & 47.85 & 6.9 & -0.658 \\
\hline PbrbZIP29 & Pbro13043.1 & Chr3 & 1131 & 22992061 & $2.3 \mathrm{E}+07$ & 5 & 41.34 & 8.92 & -0.892 \\
\hline PbrbZIP30 & Pbro13133.1 & Chr3 & 1317 & 22249661 & $2.2 \mathrm{E}+07$ & 4 & 46.86 & 6.11 & -0.799 \\
\hline PbrbZIP31 & Pbr013209.1 & Chr3 & 615 & 21719032 & $2.2 \mathrm{E}+07$ & 1 & 23.48 & 6.3 & -0.841 \\
\hline PbrbZIP32 & Pbro13267.1 & Chr3 & 1362 & 21256215 & $2.1 \mathrm{E}+07$ & 9 & 50.43 & 6.1 & -0.399 \\
\hline PbrbZIP33 & Pbro14120.1 & Chr6 & 957 & 9323825 & 9326350 & 6 & 32.7 & 5.78 & -0.679 \\
\hline PbrbZIP34 & Pbro14592.1 & Chr5 & 1350 & 22847185 & $2.3 \mathrm{E}+07$ & 5 & 49.69 & 6.3 & -0.794 \\
\hline PbrbZIP35 & Pbr014594.1 & Chr5 & 1533 & 22872574 & $2.3 \mathrm{E}+07$ & 12 & 56.13 & 6.03 & -0.531 \\
\hline PbrbZIP36 & Pbr015119.3 & Chr6 & 1002 & 19889864 & $2 E+07$ & 2 & 37.23 & 5.48 & -0.456 \\
\hline PbrbZIP37 & Pbro15675.1 & Chr2 & 1002 & 6170887 & 6178380 & 8 & 37.25 & 8.59 & -0.615 \\
\hline PbrbZIP38 & Pbr016302.1 & Chr6 & 1284 & 21111673 & $2.1 \mathrm{E}+07$ & 12 & 43.54 & 6.88 & -0.887 \\
\hline PbrbZIP39 & Pbr016568.1 & Chr17 & 609 & 17890291 & $1.8 \mathrm{E}+07$ & 1 & 23.37 & 5.9 & -0.987 \\
\hline PbrbZIP40 & Pbr017262.1 & Chr15 & 1050 & 20035048 & $2 \mathrm{E}+07$ & 11 & 36.44 & 5.53 & -0.906 \\
\hline PbrbZIP41 & Pbr017284.1 & Chr11 & 1050 & 24819436 & $2.5 \mathrm{E}+07$ & 5 & 38.31 & 9.49 & -0.798 \\
\hline PbrbZIP42 & Pbro17778.1 & Chr12 & 1320 & 20360616 & $2 \mathrm{E}+07$ & 4 & 47.73 & 8.76 & -0.641 \\
\hline PbrbZIP43 & Pbro17979.1 & Chr17 & 459 & 19768805 & $2 E+07$ & 1 & 17.64 & 9.65 & -0.756 \\
\hline PbrbZIP44 & Pbro18534.1 & Chr13 & 480 & 7268883 & 7270283 & 1 & 17.92 & 7.11 & -0.732 \\
\hline PbrbZIP45 & Pbr018536.1 & Chr13 & 429 & 7319005 & 7319433 & 1 & 15.63 & 9.45 & -0.78 \\
\hline PbrbZIP46 & Pbr018746.1 & Chr8 & 945 & 10553837 & $1.1 \mathrm{E}+07$ & 6 & 34.2 & 5.04 & -0.473 \\
\hline PbrbZIP47 & Pbro19461.1 & Chr10 & 921 & 22800599 & $2.3 \mathrm{E}+07$ & 5 & 33.89 & 8.86 & -0.759 \\
\hline PbrbZIP48 & Pbro19779.1 & Chr15 & 459 & 6992833 & 6993291 & 1 & 17.77 & 6.97 & -0.753 \\
\hline PbrbZIP49 & Pbr020210.1 & Chr6 & 465 & 4248339 & 4248803 & 1 & 17.7 & 8.04 & -0.818 \\
\hline
\end{tabular}


Table 1 (continued)

\begin{tabular}{|c|c|c|c|c|c|c|c|c|c|}
\hline Name & ID & Chr.No & ORF & Strat & End & Extron num & $M W(K D A)$ & $\mathrm{PI}$ & GRAVY \\
\hline PbrbZIP50 & Pbr020743.1 & Chr10 & 807 & 17290780 & 1.7E+ 07 & 2 & 29.31 & 6.15 & -0.686 \\
\hline PbrbZIP51 & Pbr021041.1 & Chr1 & 1272 & 3250368 & 3253437 & 12 & 45.17 & 8.69 & -0.697 \\
\hline PbrbZIP52 & Pbr022222.1 & Chr9 & 609 & 18881465 & $1.9 \mathrm{E}+07$ & 1 & 23.28 & 5.74 & -0.885 \\
\hline PbrbZIP53 & Pbr022503.1 & Chr17 & 888 & 2569875 & 2573351 & 4 & 32.79 & 6.21 & -0.726 \\
\hline PbrbZIP54 & Pbr022685.1 & Chr3 & 429 & 1149894 & 1151224 & 1 & 16.31 & 9.09 & -0.806 \\
\hline PbrbZIP55 & Pbr022894.1 & Chr2 & 2187 & 7309361 & 7312996 & 2 & 78.57 & 6.85 & -0.511 \\
\hline PbrbZIP56 & Pbr022933.1 & Chr2 & 738 & 6976334 & 6977338 & 1 & 27.18 & 7.12 & -0.783 \\
\hline PbrbZIP57 & Pbr023279.1 & Chr2 & 675 & 15827845 & $1.6 \mathrm{E}+07$ & 1 & 23.86 & 9.64 & -0.626 \\
\hline PbrbZIP58 & Pbr024746.1 & Chr2 & 909 & 8549572 & 8555592 & 2 & 33.82 & 5.67 & -0.775 \\
\hline PbrbZIP59 & Pbr025283.1 & Chr5 & 1377 & 19163038 & $1.9 \mathrm{E}+07$ & 6 & 49.73 & 6.03 & -0.676 \\
\hline PbrbZIP60 & Pbr026554.1 & Chr8 & 1143 & 4008765 & 4014636 & 4 & 42.93 & 7.24 & -0.982 \\
\hline PbrbZIP61 & Pbr026723.2 & Chr14 & 918 & 8763592 & 8766217 & 7 & 33.09 & 5.09 & -0.708 \\
\hline PbrbZIP62 & Pbr026741.1 & Chr3 & 1062 & 2967110 & 2969439 & 4 & 39.75 & 6.22 & -0.976 \\
\hline PbrbZIP63 & Pbr026913.1 & Chr15 & 1089 & 28456413 & $2.8 \mathrm{E}+07$ & 8 & 41.09 & 6.33 & -0.532 \\
\hline PbrbZIP64 & Pbr027414.1 & Chr5 & 774 & 12967287 & $1.3 \mathrm{E}+07$ & 1 & 27.88 & 5.92 & -0.621 \\
\hline PbrbZIP65 & Pbr027468.1 & Chr13 & 1572 & 2302476 & 2305727 & 10 & 58.54 & 5.98 & -0.471 \\
\hline PbrbZIP66 & Pbr027818.1 & Chr15 & 495 & 9823393 & 9826153 & 4 & 17.78 & 9 & -0.984 \\
\hline PbrbZIP67 & Pbr028080.1 & Chr8 & 1728 & 14864215 & $1.5 \mathrm{E}+07$ & 4 & 62.9 & 6.92 & -0.931 \\
\hline PbrbZIP68 & Pbr028081.1 & Chr8 & 1515 & 14868512 & $1.5 \mathrm{E}+07$ & 4 & 54.73 & 5.85 & -0.842 \\
\hline PbrbZIP69 & Pbr028249.1 & Chr12 & 486 & 5166957 & 5168690 & 4 & 18.19 & 9.12 & -0.814 \\
\hline PbrbZIP70 & Pbr028659.1 & Chr12 & 1359 & 12490432 & $1.2 \mathrm{E}+07$ & 11 & 50.2 & 8.83 & -0.646 \\
\hline PbrbZIP71 & Pbr029239.1 & Chr1 & 459 & 2389878 & 2390653 & 1 & 17.64 & 9.65 & -0.756 \\
\hline PbrbZIP72 & Pbr029701.1 & Chr9 & 1044 & 14021920 & $1.4 \mathrm{E}+07$ & 11 & 36.27 & 5.74 & -0.887 \\
\hline PbrbZIP73 & Pbr030038.1 & Chr13 & 615 & 4056908 & 4057801 & 1 & 23.47 & 5.93 & -0.751 \\
\hline PbrbZIP74 & Pbr030476.1 & Chr5 & 861 & 2296058 & 2297044 & 2 & 30.98 & 5.12 & -0.585 \\
\hline PbrbZIP75 & Pbr030604.1 & Chr9 & 1341 & 19283974 & $1.9 \mathrm{E}+07$ & 10 & 49.78 & 7.28 & -0.65 \\
\hline PbrbZIP76 & Pbr030829.1 & Chr11 & 426 & 565147 & 566497 & 1 & 16.24 & 6.75 & -0.791 \\
\hline PbrbZIP77 & Pbr031203.1 & Chr15 & 1053 & 39848596 & $4 \mathrm{E}+07$ & 4 & 36.78 & 6.61 & -0.789 \\
\hline PbrbZIP78 & Pbr033760.1 & Chr15 & 603 & 30168150 & $3 E+07$ & 1 & 22.97 & 10.51 & -0.659 \\
\hline PbrbZIP79 & Pbr034805.1 & Chr13 & 846 & 14373472 & $1.4 \mathrm{E}+07$ & 3 & 30.55 & 9.65 & -0.723 \\
\hline PbrbZIP80 & Pbr035554.1 & Chr5 & 369 & 15980857 & $1.6 \mathrm{E}+07$ & 3 & 14.03 & 9.38 & -0.475 \\
\hline PbrbZIP81 & Pbr035863.1 & Chr12 & 1338 & 16253048 & $1.6 \mathrm{E}+07$ & 10 & 49.71 & 6.98 & -0.642 \\
\hline PbrbZIP82 & Pbr036339.1 & Chr10 & 1026 & 19091541 & $1.9 E+07$ & 4 & 37.35 & 6.6 & -0.825 \\
\hline PbrbZIP83 & Pbr036605.1 & Chr11 & 1539 & 18152763 & $1.8 \mathrm{E}+07$ & 11 & 56.54 & 6.75 & -0.53 \\
\hline PbrbZIP84 & Pbr037165.1 & Chr16 & 1230 & 19058863 & $1.9 \mathrm{E}+07$ & 12 & 43.64 & 6.62 & -0.769 \\
\hline PbrbZIP85 & Pbr038249.1 & Chr11 & 1056 & 4628100 & 4630519 & 4 & 39.69 & 6.27 & -0.977 \\
\hline PbrbZIP86 & Pbr039911.1 & scaffold868.0 & 1134 & 46463 & 50532 & 4 & 42.23 & 7.2 & -0.893 \\
\hline PbrbZIP87 & Pbr039916.1 & scaffold868.0 & 1134 & 103303 & 107372 & 4 & 42.23 & 7.2 & -0.893 \\
\hline PbrbZIP88 & Pbr040390.1 & scaffold888.0 & 1368 & 59641 & 62060 & 4 & 49.53 & 9.43 & -0.585 \\
\hline PbrbZIP89 & Pbr040479.1 & Chr2 & 474 & 15517927 & $1.6 \mathrm{E}+07$ & 1 & 17.9 & 5.41 & -0.794 \\
\hline PbrbZIP90 & Pbr041663.1 & Chr7 & 1002 & 2182780 & 2189697 & 8 & 17.9 & 5.41 & -0.794 \\
\hline PbrbZIP91 & Pbr042765.1 & Chr10 & 807 & 17608035 & $1.8 \mathrm{E}+07$ & 2 & 29.34 & 6.15 & -0.695 \\
\hline PbrbZIP92 & Pbr042848.1 & Chr8 & 780 & 6290536 & 6291676 & 1 & 27.28 & 5.85 & -0.673 \\
\hline
\end{tabular}

PbrbZIP21, and PbrbZIP92 (subgroup UN; Fig. 1) formed three small, unique subgroups in the phylogenetic tree and might have evolutionary trajectories unrelated to other subgroups. Subgroup $S$ had the largest number of PbrbZIP genes (17 genes), followed by subgroups I (14 genes) and A (11 genes). Subgroup K had only one gene (PbrbZIP36).

The potential function of these genes could be depicted based on the annotation information of Gene Ontology (GO) and Kyoto Encyclopedia of Genes and Genomes 


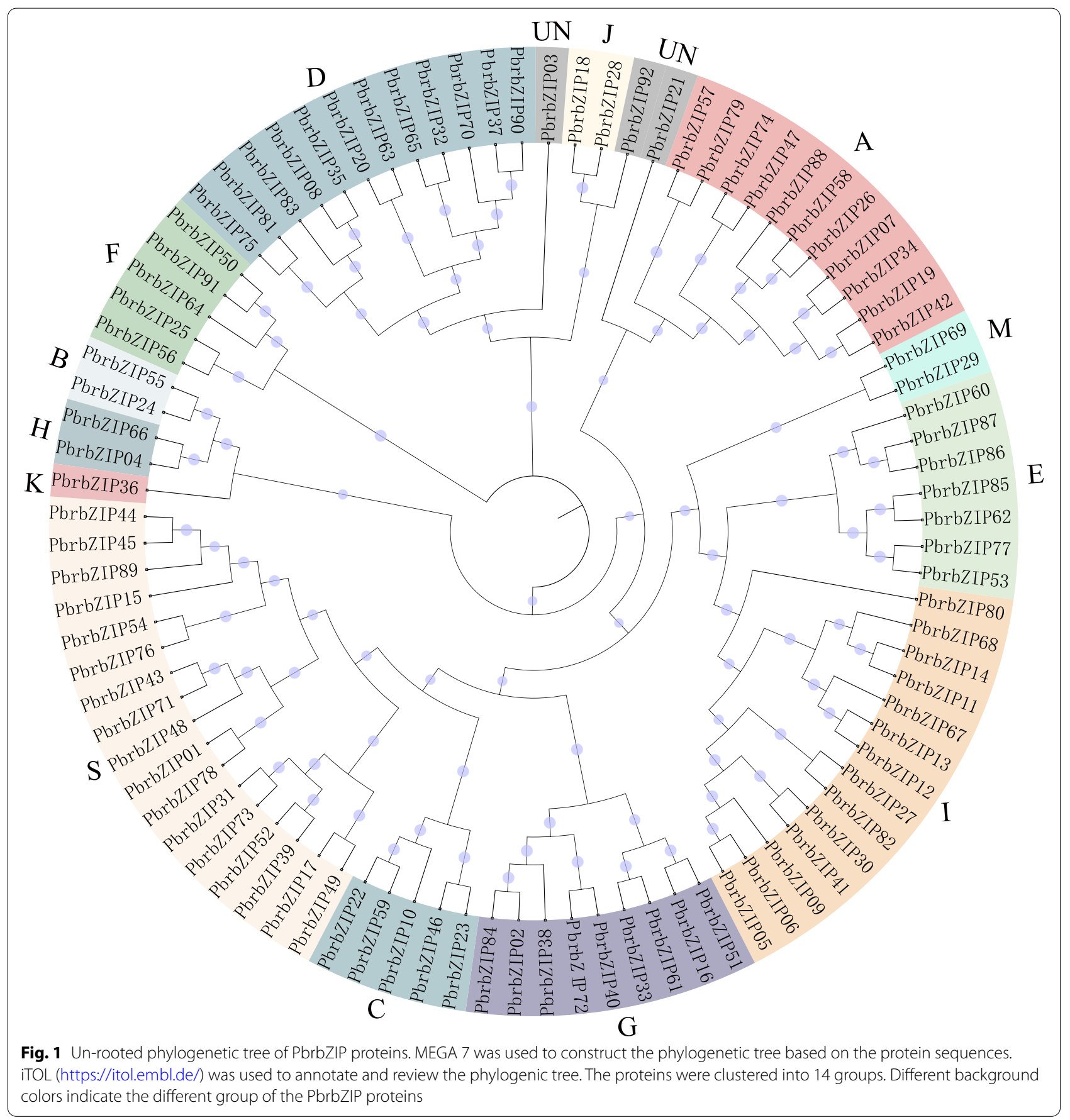

(KEGG) databases. Functional enrichment analysis was performed to forecast the potential functions of PbrbZIP genes. PbrbZIP genes were enriched mainly in transcription regulator activity, molecular function, DNA-binding TF activity, biosynthetic process, and some regulatory functions; the regulation of the expression of TFs was closely related to all of these functions (Fig. S2a). In addition, the KEGG enrichment result showed that these genes were enriched only in plant hormone signal transduction, circadian rhythm, and mitogen-activated protein kinase (MAPK) signaling, and these mechanisms were related mainly to the regulation of downstream gene expression by bZIP family TFs (Fig. S2b). The crucial TFs of these pathways were also discovered through BLASTP. ATbZIP56 (HY5), whose orthologous genes were PbrbZIP66 and PbrbZIP04, integrated hormonal signaling 


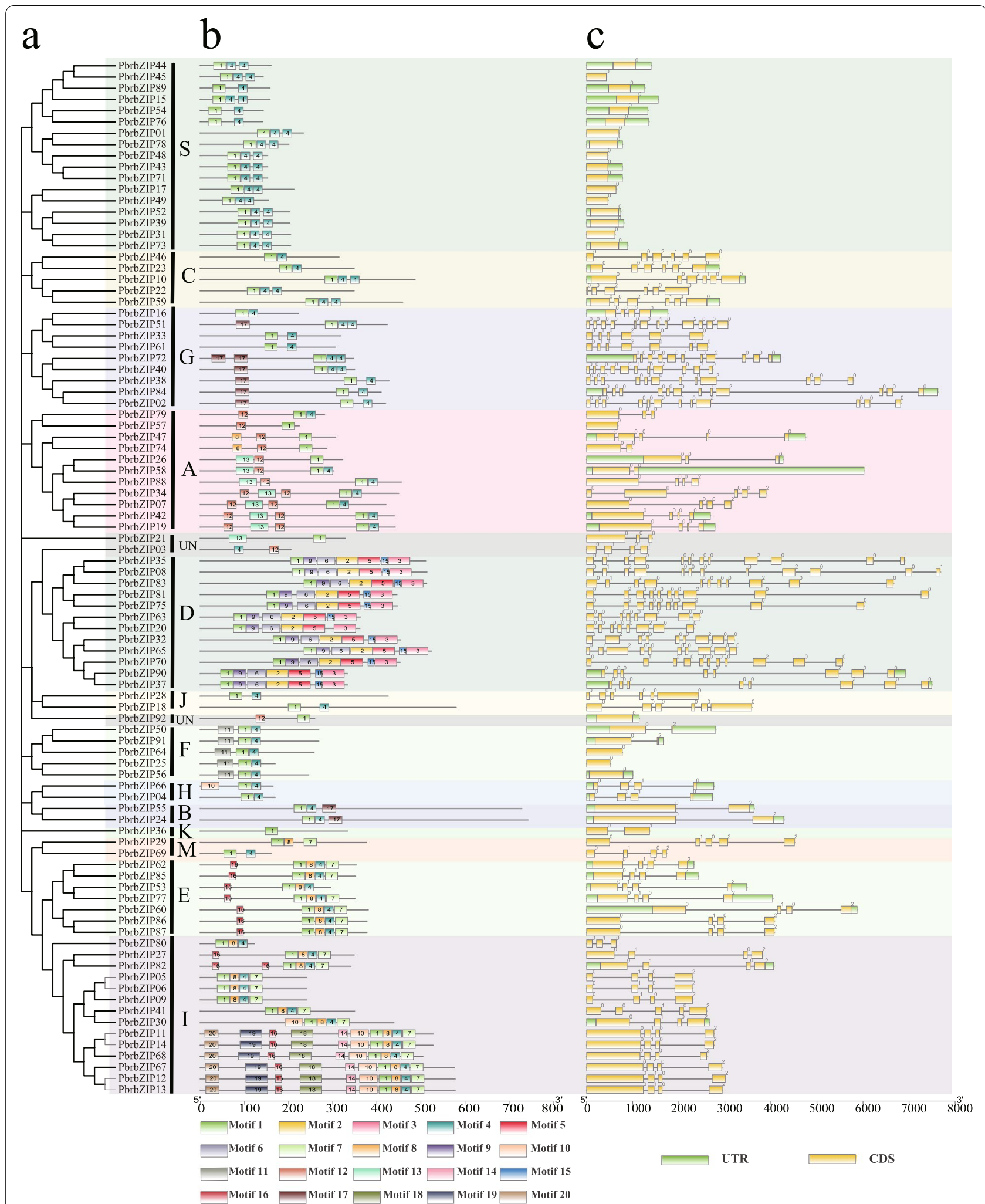

Fig. 2 Gene structure schematics and preserved motifs patterns in the PbrbZIP family. a Subgroup classification: The phylogenetic tree was derived from 92 PbrbZIP genes with MEGA 7. b Conserved motif analysis: 20 separate patterns were identified with the MEME suite and each pattern was depicted with different colors. c Gene structural analysis 
pathways (auxin, gibberellin, brassinolide, and ethylene) and interacted with the promoter of the monoterpene synthase gene QH6 in modulating its rhythmic expression [34, 35]. ATbZIP51 (the ortholog of PbrbZIP82 and PbrbZIP27) regulated the immune signaling of plants downstream of the MPK3 signal transduction pathway [36].

\section{Conserved motif and structure analyses of pear bZIP proteins}

Given that the structure of exon and intron can provide important evidence supporting the phylogenetic relationships of a gene family [37], a rootless phylogenetic tree was established to analyze the evolutionary history of the PbrbZIP gene family by multi-sequence alignment (Fig. 2a). In this study, an online program of Multiple Expectation Maximization for Motif Elicitation (MEME) was used to detect motif patterns. As shown in Fig. 2b, 20 preserved patterns, including the bZIP domain (motif \#1 and motif \#4), were identified, and their multilevel pattern amino acid consensus sequences are listed in Table S2. The proteins categorized within the same group tended to share a similar motif composition, but varied significantly between groups, which further supported the group definitions. As shown in Fig. 2b, among PbrbZIPs, motif \#1, containing a basic DNA-binding domain, which belonged to a typical bZIP domain, was detected in all members as a conserved pattern, except PbrbZIP03. Some patterns were present only in specific subgroups, including motif \#6 in subgroups I and E; motif \#7 in subgroups C, I, S, E, F, G, and M; and motif \#17 in subgroups $A$ and UN, except PbrbZIP03. However, some unique patterns could be detected only in specific subgroups. For instance, the pattern $[\# 2,3,5,8,19]$ in subgroup D, pattern $[\# 9,10,12,13,14,18]$ in subgroup I, pattern \#11 in subgroup $F$, pattern \#16 in subgroup $A$, and pattern \#20 in subgroup G. Many subgroups were composed of certain patterns, but huge differences were found among subgroups. According to the results of gene structure analysis, the number of exons and the gene structure of the PbrbZIP gene family were diverse (Fig. 2c). As shown in Fig. 2c, 22 bZIP genes were identified with no introns, all of which belonged to subgroups S, F, and PbrbZIP92, and which accounted for $23.6 \%$ of the total number of PbrbZIP genes. Among the intron-containing genes, the number of introns in open reading frames ranged from 0 to 11 , and the number of introns in different groups varied greatly. For example, a greater degree of variation in the number of introns occurred in subgroups A, D, and G, ranging from 0 to 4,7 to 11 , and 3 to 11 , respectively. However, the number of introns in the remaining groups was smaller, for example, three in subgroups $\mathrm{E}$ and $\mathrm{H}$ and three to four in subgroup I. As a result, we proposed that exon loss and gain occurred during the evolution of $\mathrm{Pbr}$ $b Z I P$ genes, and the evolution and division among different subgroups might occur at an early stage.

\section{Evolutionary and phylogenetic relationship of PbrbZIP genes}

An intragenomic synteny analysis was performed to understand the evolutionary process of PbrbZIP genes, and conservation chromosome blocks were identified in Chinese white pears. In Fig. 3, the landscape of ortholog PbrbZIP gene pairs showed that the chromosomal distribution was random. WGD/segmental duplication, tandem duplication, and transposition events are the major causes of gene family expansion and affect the evolution of protein-coding gene families [38]. In this study, duplication events were detected in the $b Z I P$ gene family, and each gene was assigned to one of five different types of duplications: singleton, dispersed, proximal, tandem, and WGD/segmental through running the MCScanX package. Five types of duplications were all detected causing PbrbZIP genes to expand (Tables 2 and S3). The results showed that 65 genes $(78.31 \%)$ of the bZIP gene family of Chinese white pears were duplicated and preserved from segmental/WGD events, and almost 12 PbrbZIP genes (14.46\%) belonged to the dispersed type.

The Ks value (synonymous substitutions per site) could be used to estimate the dates of WGD and segmental duplication [39].. Previous studies showed that two genome-wide replication events occurred in the pear genome: the ancient WGD occurred in $\sim 140$ million years ago (MYA) and the modern WGD occurred in 30-45years of MYA [33, 40]. The Ks values were used to estimate the evolutionary date of gene duplication events in the PbrbZIP gene family. As seen in the replication period estimated by Ks values in Table 3, most of the PbrbZIP genes were around the recent WGD event, and some were in the ancient WGD. The ratio of nonsynonymous substitutions per nonsynonymous site (Ka) to Ks was also used to predict the selection pressure of duplicated genes: $\mathrm{Ka} / \mathrm{Ks}>1$ meant positive selection, $\mathrm{Ka} /$ $\mathrm{Ks}=1$ meant neutral selection, and $\mathrm{Ka} / \mathrm{Ks}<1$ denoted purification (negative) selection [41]. The $\mathrm{Ka} / \mathrm{Ks}$ ratio of all PbrbZIP genes was lower than 1, indicating that $P b r$ $b Z I P$ genes evolved mainly under purifying selection.

\section{Expression patterns of PbrbZIP genes in response to cold stress}

The bZIP proteins might be related to cold and drought stresses in plants [22, 42, 43]. However, limited information regarding the response of bZIP TFs to drought and cold stresses has been reported in Chinese white pears. The response of pears to drought and low-temperature 


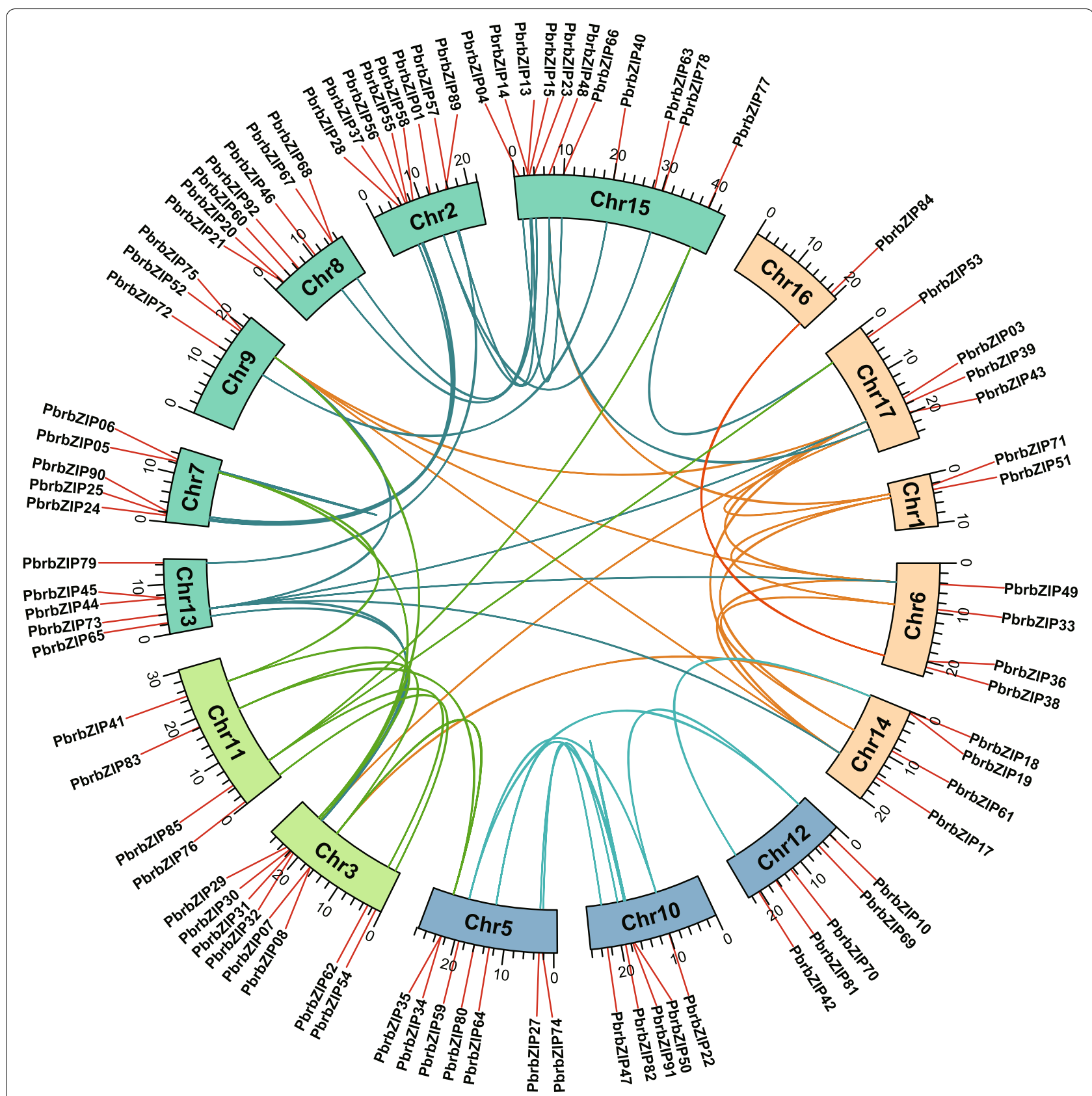

Fig. 3 Distribution and collinearity of PbrbZIPs. The lines in various colors within the circle indicate collinearity relationships between PbrbZIP genes. The red lines along the circumference of the circle show the location of genes on chromosomes

Table 2 Numbers of bZIP genes from different origins in pear (Pyrus bretschneideri)

\begin{tabular}{llllll}
\hline Duplication type & Singleton & Dispersed & Proximal & Tandem & WGD/segmental \\
\hline No. of bZIP genes from different origins (percentage) & $1(1.20)$ & $12(14.46)$ & $2(2.41)$ & $3(3.61)$ & $65(78.31)$
\end{tabular}


Table 3 The duplicate mode and estimation of absolute date for large-scale duplication events for PbrbZIPs

\begin{tabular}{|c|c|c|c|c|c|c|c|c|}
\hline \multirow[t]{2}{*}{ Method } & \multicolumn{2}{|c|}{ Colinearity gene pairs } & \multicolumn{2}{|c|}{ Duplication type } & \multirow[t]{2}{*}{$\mathrm{Ka}$} & \multirow[t]{2}{*}{ Ks } & \multirow[t]{2}{*}{$\mathrm{Ka} / \mathrm{Ks}$} & \multirow[t]{2}{*}{ MYA } \\
\hline & Gene1 & Gene2 & Gene1 & Gene2 & & & & \\
\hline NG & PbrbZIP51 & PbrbZIP61 & WGD & WGD & 0.52 & 2 & 0.26 & 669.26 \\
\hline NG & PbrbZIP71 & PbrbZIP48 & WGD & WGD & 0.08 & 0.11 & 0.72 & 37.1 \\
\hline NG & PbrbZIP71 & PbrbZIP43 & WGD & WGD & $\mathrm{Na}$ & $\mathrm{Na}$ & $\mathrm{Na}$ & $\mathrm{Na}$ \\
\hline NG & PbrbZIP51 & PbrbZIP33 & WGD & WGD & 0.56 & 2.83 & 0.2 & 947.37 \\
\hline NG & PbrbZIP51 & PbrbZIP16 & WGD & NA & 0.07 & 0.23 & 0.29 & 78.55 \\
\hline NG & PbrbZIP50 & PbrbZIP91 & WGD & WGD & 0 & 0.02 & 0.07 & 7.81 \\
\hline NG & PbrbZIP22 & PbrbZIP10 & WGD & WGD & 0.46 & 1.74 & 0.26 & 581.6 \\
\hline NG & PbrbZIP22 & PbrbZIP59 & WGD & WGD & 0.06 & 0.25 & 0.22 & 85.02 \\
\hline NG & PbrbZIP91 & PbrbZIP64 & WGD & WGD & 0.05 & 0.14 & 0.35 & 47.99 \\
\hline NG & PbrbZIP82 & PbrbZIP27 & WGD & WGD & 0.07 & 0.19 & 0.35 & 63.45 \\
\hline NG & PbrbZIP47 & PbrbZIP74 & WGD & WGD & 0.09 & 0.23 & 0.38 & 78.38 \\
\hline NG & PbrbZIP50 & PbrbZIP64 & WGD & WGD & 0.05 & 0.11 & 0.42 & 38.21 \\
\hline NG & PbrbZIP85 & PbrbZIP77 & WGD & WGD & 0.34 & $\mathrm{Na}$ & $\mathrm{Na}$ & $\mathrm{Na}$ \\
\hline NG & PbrbZIP85 & PbrbZIP53 & WGD & WGD & 0.35 & $\mathrm{Na}$ & $\mathrm{Na}$ & $\mathrm{Na}$ \\
\hline NG & PbrbZIP41 & PbrbZIP30 & WGD & WGD & 0.05 & 0.22 & 0.24 & 74.34 \\
\hline NG & PbrbZIP76 & PbrbZIP54 & WGD & WGD & 0.03 & 0.16 & 0.15 & 54.99 \\
\hline NG & PbrbZIP85 & PbrbZIP62 & WGD & WGD & 0.03 & 0.24 & 0.14 & 80.94 \\
\hline NG & PbrbZIP83 & PbrbZIP08 & WGD & WGD & 0.03 & 0.16 & 0.22 & 53.83 \\
\hline NG & PbrbZIP83 & PbrbZIP35 & WGD & WGD & 0.04 & 0.15 & 0.24 & 50.18 \\
\hline NG & PbrbZIP41 & PbrbZIP05 & WGD & WGD & 0.25 & 1.55 & 0.16 & 519.43 \\
\hline NG & PbrbZIP42 & PbrbZIP19 & WGD & WGD & 0.05 & 0.16 & 0.3 & 53.19 \\
\hline NG & PbrbZIP10 & PbrbZIP59 & WGD & WGD & 0.5 & 1.61 & 0.31 & 539.82 \\
\hline NG & PbrbZIP73 & PbrbZIP17 & WGD & WGD & 0.64 & 2.57 & 0.25 & 859.53 \\
\hline NG & PbrbZIP73 & PbrbZIP39 & WGD & WGD & 0.34 & 1.55 & 0.22 & 518.94 \\
\hline NG & PbrbZIP79 & PbrbZIP57 & WGD & WGD & 0.12 & 0.19 & 0.64 & 62.31 \\
\hline NG & PbrbZIP65 & PbrbZIP32 & WGD & WGD & 0.06 & 0.25 & 0.23 & 82.12 \\
\hline NG & PbrbZIP73 & PbrbZIP31 & WGD & WGD & 0.05 & 0.26 & 0.19 & 88.16 \\
\hline NG & PbrbZIP73 & PbrbZIP49 & WGD & WGD & 0.57 & 1.76 & 0.33 & 588.3 \\
\hline NG & PbrbZIP73 & PbrbZIP52 & WGD & WGD & 0.36 & 1.42 & 0.25 & 476.29 \\
\hline NG & PbrbZIP17 & PbrbZIP39 & WGD & WGD & 0.62 & $\mathrm{Na}$ & $\mathrm{Na}$ & $\mathrm{Na}$ \\
\hline NG & PbrbZIP19 & PbrbZIP07 & WGD & WGD & 0.5 & $\mathrm{Na}$ & $\mathrm{Na}$ & $\mathrm{Na}$ \\
\hline NG & PbrbZIP17 & PbrbZIP49 & WGD & WGD & 0.15 & 0.3 & 0.49 & 98.8 \\
\hline NG & PbrbZIP61 & PbrbZIP33 & WGD & WGD & 0.11 & 0.21 & 0.52 & 71.39 \\
\hline NG & PbrbZIP17 & PbrbZIP52 & WGD & WGD & 0.6 & 2.47 & 0.25 & 824.95 \\
\hline NG & PbrbZIP04 & PbrbZIP66 & WGD & WGD & 0.01 & 0.01 & 0.9 & 3 \\
\hline NG & PbrbZIP48 & PbrbZIP43 & WGD & WGD & 0.08 & 0.11 & 0.72 & 37.1 \\
\hline NG & PbrbZIP77 & PbrbZIP53 & WGD & WGD & 0.1 & 0.31 & 0.34 & 102.63 \\
\hline NG & PbrbZIP78 & PbrbZIP01 & WGD & WGD & 0.08 & 0.2 & 0.38 & 67.79 \\
\hline NG & PbrbZIP48 & PbrbZIP01 & WGD & WGD & 0.61 & 1.14 & 0.54 & 381 \\
\hline NG & PbrbZIP15 & PbrbZIP89 & WGD & WGD & 0.27 & 1.57 & 0.17 & 524.43 \\
\hline NG & PbrbZIP13 & PbrbZIP67 & WGD & WGD & 0.03 & 0.2 & 0.13 & 66.97 \\
\hline NG & PbrbZIP23 & PbrbZIP46 & WGD & WGD & 0.09 & 0.23 & 0.38 & 77.05 \\
\hline NG & PbrbZIP40 & PbrbZIP72 & WGD & WGD & 0.03 & 0.19 & 0.17 & 62.26 \\
\hline NG & PbrbZIP13 & PbrbZIP11 & WGD & NA & 0.07 & 0.31 & 0.23 & 102.58 \\
\hline NG & PbrbZIP84 & PbrbZIP38 & WGD & WGD & 0.04 & 0.16 & 0.21 & 55.06 \\
\hline NG & PbrbZIP39 & PbrbZIP31 & WGD & WGD & 0.31 & 1.44 & 0.22 & 481.17 \\
\hline NG & PbrbZIP39 & PbrbZIP49 & WGD & WGD & 0.52 & $\mathrm{Na}$ & $\mathrm{Na}$ & $\mathrm{Na}$ \\
\hline NG & PbrbZIP39 & PbrbZIP52 & WGD & WGD & 0.06 & 0.2 & 0.29 & 66.62 \\
\hline
\end{tabular}


Table 3 (continued)

\begin{tabular}{|c|c|c|c|c|c|c|c|c|}
\hline \multirow[t]{2}{*}{ Method } & \multicolumn{2}{|c|}{ Colinearity gene pairs } & \multicolumn{2}{|c|}{ Duplication type } & \multirow[t]{2}{*}{ Ka } & \multirow[t]{2}{*}{ Ks } & \multirow[t]{2}{*}{$\mathrm{Ka} / \mathrm{Ks}$} & \multirow[t]{2}{*}{ MYA } \\
\hline & Gene1 & Gene2 & Gene1 & Gene2 & & & & \\
\hline NG & PbrbZIP37 & PbrbZIP90 & WGD & WGD & 0.01 & 0.14 & 0.09 & 45.83 \\
\hline NG & PbrbZIP56 & PbrbZIP25 & WGD & WGD & 0.1 & 0.24 & 0.42 & 81.4 \\
\hline NG & PbrbZIP55 & PbrbZIP24 & WGD & WGD & 0.05 & 0.21 & 0.26 & 69.07 \\
\hline NG & PbrbZIP58 & PbrbZIP26 & dispersed & NA & 0.04 & 0.14 & 0.27 & 47.62 \\
\hline NG & PbrbZIP08 & PbrbZIP35 & WGD & WGD & 0 & 0.01 & 0.2 & 2.81 \\
\hline NG & PbrbZIP07 & PbrbZIP34 & WGD & WGD & 0.01 & 0.01 & 1.09 & 3.77 \\
\hline NG & PbrbZIP30 & PbrbZIP05 & WGD & WGD & 0.26 & 1.74 & 0.15 & 581.11 \\
\hline NG & PbrbZIP31 & PbrbZIP52 & WGD & WGD & 0.34 & 1.35 & 0.25 & 450.63 \\
\hline NG & PbrbZIP49 & PbrbZIP52 & WGD & WGD & 0.58 & $\mathrm{Na}$ & $\mathrm{Na}$ & $\mathrm{Na}$ \\
\hline NG & PbrbZIP05 & PbrbZIP06 & WGD & WGD & $\mathrm{Na}$ & $\mathrm{Na}$ & $\mathrm{Na}$ & $\mathrm{Na}$ \\
\hline NG & PbrbZIP06 & PbrbZIP09 & WGD & NA & 0.02 & 0.15 & 0.11 & 48.73 \\
\hline NG & PbrbZIP05 & PbrbZIP09 & WGD & WGD & 0.02 & 0.15 & 0.11 & 48.73 \\
\hline NG & PbrbZIP67 & PbrbZIP11 & WGD & WGD & 0.06 & 0.34 & 0.19 & 114.28 \\
\hline NG & PbrbZIP60 & PbrbZIP86 & dispersed & NA & 0.03 & 0.08 & 0.36 & 26.09 \\
\hline NG & PbrbZIP86 & PbrbZIP87 & WGD & NA & $\mathrm{Na}$ & $\mathrm{Na}$ & $\mathrm{Na}$ & $\mathrm{Na}$ \\
\hline
\end{tabular}

stresses was studied by analyzing the transcriptome data of PbrbZIP genes (Figs. 4a and 5a).

In Fig. 4a, cluster A contained eight PbrbZIP genes that were significantly upregulated after cold treatment at $5 \mathrm{~h}$ post treatment (hpt) and $12 \mathrm{hpt}$. Cluster $\mathrm{C}$ with three genes experienced upregulation at $5 \mathrm{hpt}$ and then began a downregulation between $12 \mathrm{hpt}$ and $24 \mathrm{hpt}$. Again, 19 genes of cluster D were highly induced from $5 \mathrm{hpt}$ to 24 hpt. Most genes of clusters E, F, and G were highly expressed at $0 \mathrm{hpt}$ and downregulated at $5 \mathrm{hpt}$, but then upregulated again later. Most genes in cluster B were not significantly induced by cold treatment at all tested times. Twenty-three genes that were at least 1.5 times more regulated after cold treatment were chosen to investigate their expression patterns (Table S4). Six genes were found in subgroup $I$, five genes in subgroup $S$, three genes in A and G, and two genes in C and F; PbrbZIP36 belonging to subgroup $\mathrm{K}$ were upregulated under cold stress. The expression levels of 19 genes in cluster D were higher than those in other clusters, and most genes in cluster B belonged to $\mathrm{S}, \mathrm{G}$, I, and F subgroups. These results indicated that genes of A, C, G, I, and S subgroups mainly mediated cold stress responses by taking part in biological pathways.

Quantitative real-time polymerase chain reaction (qRTPCR) were performed to analyze the relative transcript abundance of six selected genes so as to confirm whether the expression of these genes differed under low-temperature stress. As shown in Fig. 4b, PbrbZIP54, PbrbZIP76, and PbrbZIP89 from subgroup $S$ were upregulated at 2 hpt but downregulated at $3 \mathrm{hpt}$, and upregulated again at 12 hpt. PbrbZIP16 and PbrbZIP51 in subgroup G were upregulated at 6 and $24 \mathrm{hpt}$ but downregulated at $12 \mathrm{hpt}$. PbrbZIP59 belonging to subgroup $C$ was upregulated only at $3 \mathrm{hpt}$ after cold treatment and then downregulated. These results closely matched the RNA-seq data.

\section{Expression patterns of PbrbZIP genes in response to drought stress}

The same approach was used to analyze bZIP TFs in response to drought stress. As shown in Fig. 5a, cluster A (four genes) was significantly downregulated after drought treatment for 1 and $3 \mathrm{hpt}$ and upregulated at $6 \mathrm{hpt}$. Cluster B contained 24 PbrbZIP genes that were upregulated at $6 \mathrm{hpt}$. In cluster C, 14 PbrbZIP genes were downregulated at $3 \mathrm{hpt}$ and $6 \mathrm{hpt}$. Cluster D contained five genes upregulated at $1 \mathrm{hpt}$ after drought treatment. Cluster E (22 genes) had no apparent differences in expression in response to drought stress. Cluster F contained two genes that were significantly upregulated at 1 and $6 \mathrm{hpt}$, but downregulated at $3 \mathrm{hpt}$. Four genes in cluster $\mathrm{G}$ were upregulated at 3 hpt, but downregulated at $6 \mathrm{hpt}$ under drought stress. The genes belonging to cluster $\mathrm{H}$ had relatively high expression from 0 to $3 \mathrm{hpt}$, but downregulated at $6 \mathrm{hpt}$. Nineteen genes, which were upregulated at least twofold under drought stress, were selected for a further survey of their expression patterns (Table S4). Five genes in group I, four genes in group $\mathrm{S}$, three genes in group $\mathrm{C}$, two genes in $\mathrm{A}$ and $\mathrm{G}$, and one gene in $\mathrm{B}, \mathrm{F}$, and $\mathrm{K}$ were upregulated under drought stress. Compared with the genes in other clusters, seven genes in cluster B and two genes in cluster A were more significantly upregulated at $3 \mathrm{hpt}$. Three genes 
a

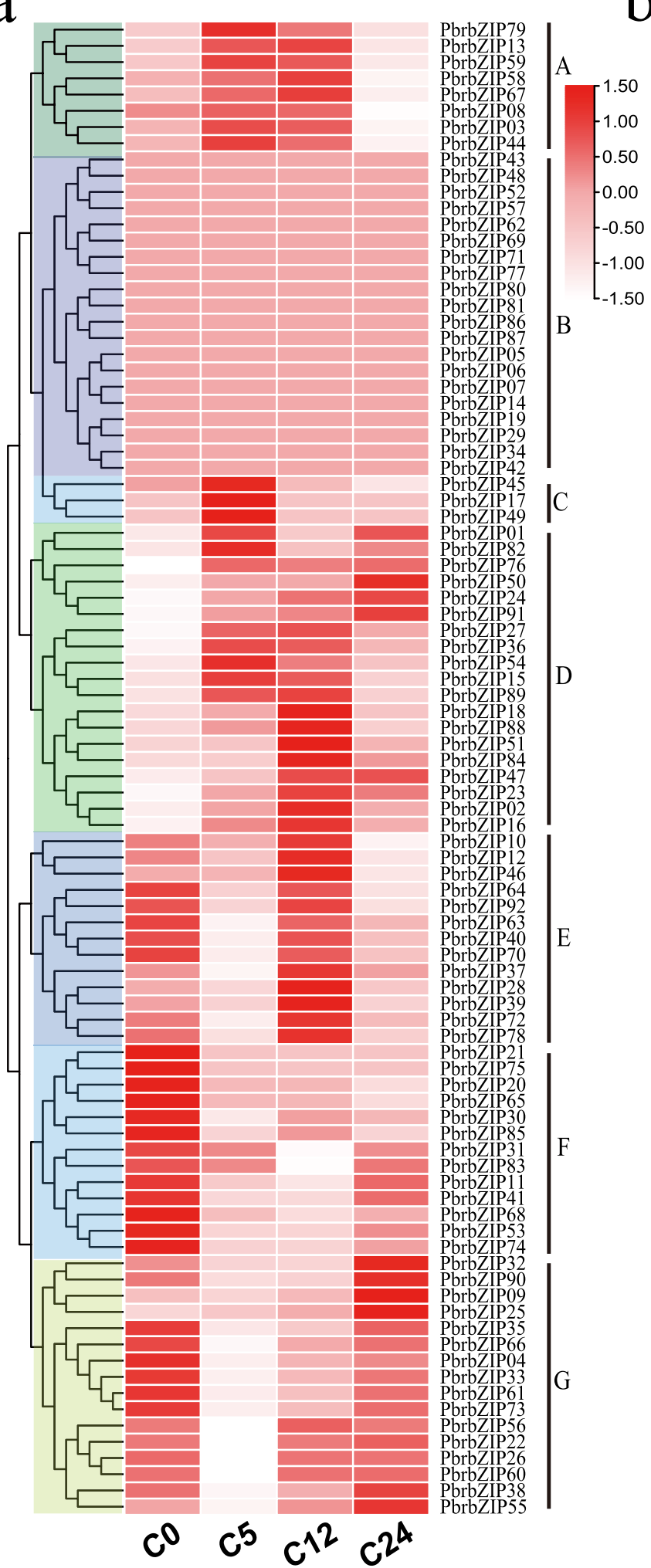

b
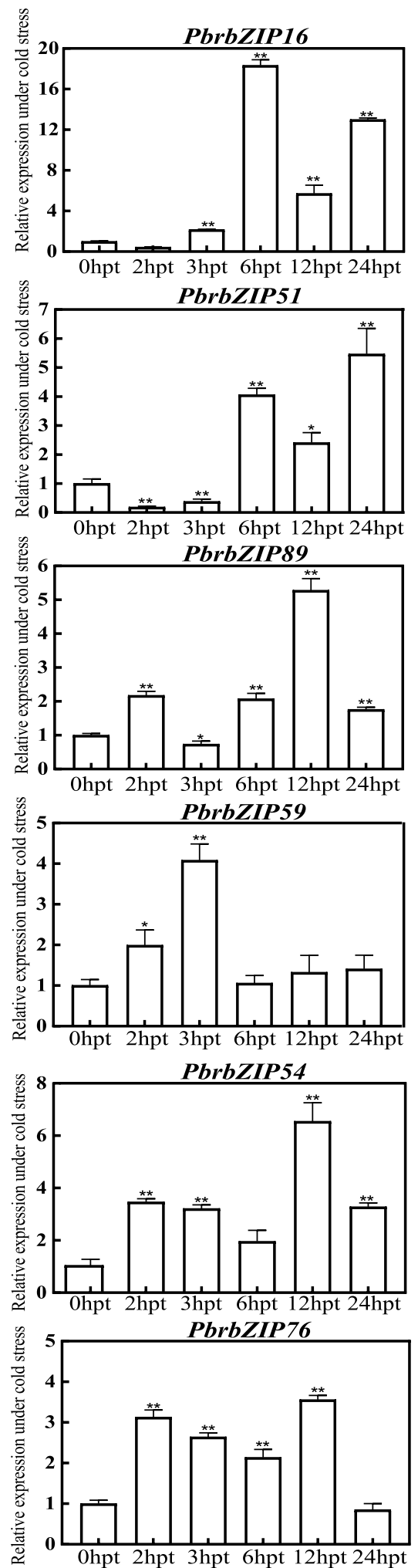

Fig. 4 Expression profile and expression analysis of PbrbZIPs under cold stress. a Expression profile of PbrbZIPs under cold stress; $\mathbf{b}$ Relative expression of PbrbZIP16, PbrbZIP51, PbrbZIP89, PbrbZIP59, PbrbZIP54 and PbrbZIP76 with cold treatment. The pear tubulin was used as internal reference for the normalization. The statistical analyses were performed using student's t-test $\left({ }^{*} p<0.05,{ }^{* *} p<0.01\right)$ 
a

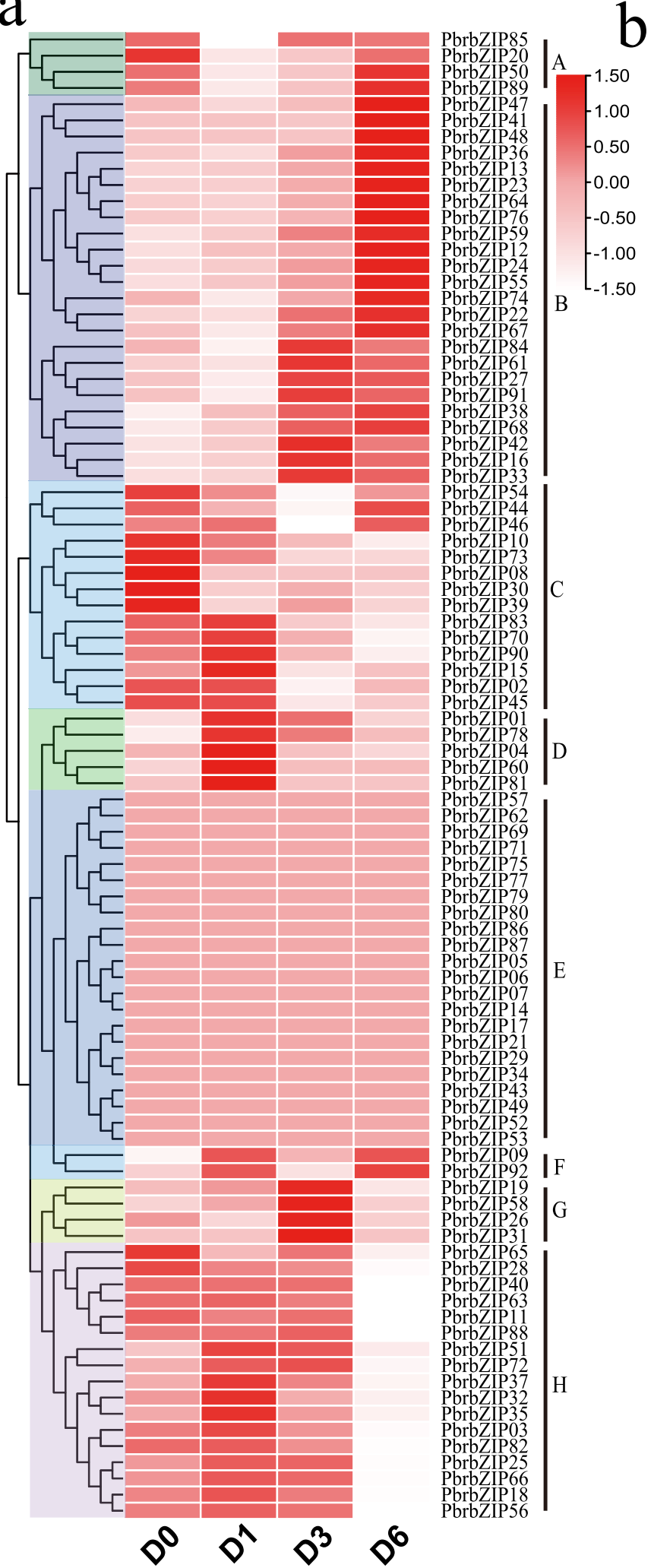

b
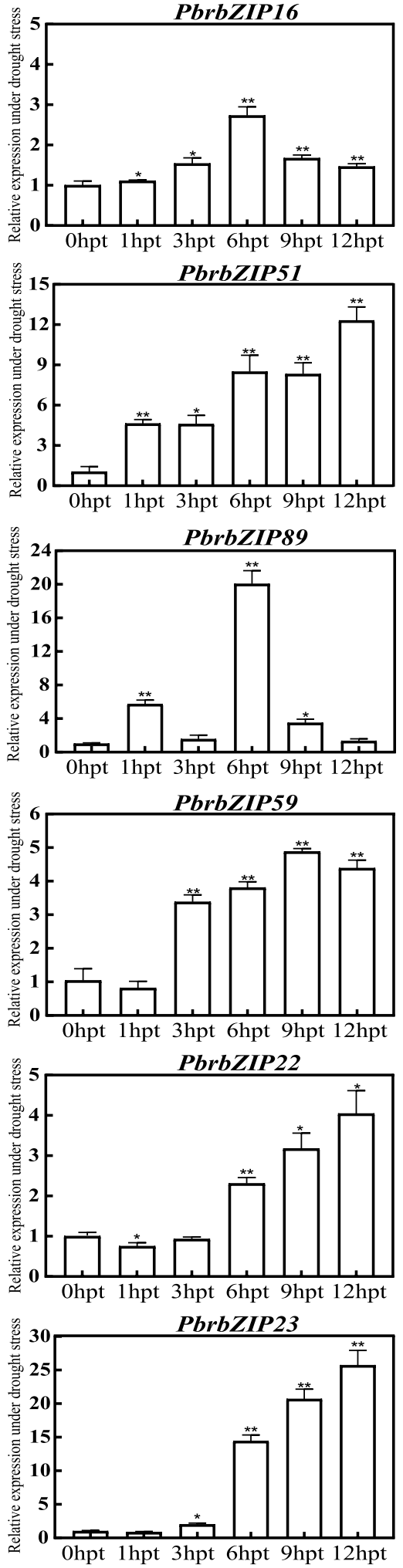

Fig. 5 Expression profile and analysis of PbrbZIPs under drought stress. a Expression profile of PbrbZIPs under drought stress; b Relative expression PbrbZIP16, PbrbZIP51, PbrbZIP89, PbrbZIP59, PbrbZIP22 and PbrbZIP23 with drought treatment. The pear tubulin was used as internal reference for the normalization. The statistical analyses were performed using student's t-test $\left({ }^{*} p<0.05,{ }^{* *} p<0.01\right)$ 
belonged to subgroup $C$, two genes to subgroup $G$, and one gene to subgroups $\mathrm{B}, \mathrm{K}$, and $\mathrm{S}$. Therefore, the PbrbZIP genes from these subgroups might be involved in some biological processes to improve the drought tolerance of pears. Meanwhile, seven genes, including PbrbZIP89, PbrbZIP76, PbrbZIP36, PbrbZIP16, PbrbZIP51, PbrbZIP59, and Pbr$b Z I P 24$, were found to be strongly upregulated after drought treatment as well as under cold treatment. The qRT-PCR results closely matched the RNA-seq data of this study (Fig. 5b). The expression of all chosen genes peaked at 6,9 , and $12 \mathrm{hpt}$, and then began to decline. These results indicated that all the aforementioned genes had a significant response to drought and cold temperature treatment. Moreover, the expression patterns of PbrbZIP16 and Pbr$b Z I P 51$ indicated that these genes were involved in stress resistance and specific genes might have different response patterns under different stresses.

\section{Silencing PbrbZIP51 in P. betulaefolia provided sensitivity to drought stress}

As a significantly upregulated gene under both cold and drought stresses, PbrbZIP51 was selected to perform virus-induced gene silencing (VIGS) to further explore the role of PbrbZIP genes in drought tolerance. As shown in Fig. 6a-d, VIGS plants (p-TRV1 and p-TRV2) suffered more severe damage than control after drought treatment for 15 days. As shown in Fig. 6e and $\mathrm{f}$, the expression of PbrbZIP51 was suppressed in silenced seedlings. The electrolyte leakage (EL) (Fig. 6b) and malondialdehyde (MDA) Fig. 6c) concentrations were significantly higher in silenced pear seedlings than in control seedlings. Chl fluorescence in silenced plants was suppressed, with significantly lower $\mathrm{Fv} / \mathrm{Fm}$ ratio and Chl content compared with that in control plants (Fig. $6 \mathrm{~g}-\mathrm{i}$ ). Furthermore, quantitative measurements of the $\mathrm{H}_{2} \mathrm{O}_{2}$ content showed that the $\mathrm{H}_{2} \mathrm{O}_{2}$ content of the silenced plants was much higher than those of the control plants (Fig. 6j). These results suggested that the PbrbZIP51 gene was silenced to enhance the sensitivity to drought in $P$. betulaefollia.

\section{Discussion}

Cold and drought stresses are two of the most important limiting environmental factors that can seriously impair crop productivity. TFs play an important role in protecting plants from stress-related damage by regulating the expression levels of downstream target genes
[44]. Therefore, genetic engineering of TFs involved in stress resistance has been proposed to be a robust strategy for improving the stress tolerance of crop plants $[45,46]$. Since the release of genome sequencing data from Chinese white pears, many $T F$ genes have been identified and characterized at the genome-wide level, such as NAC TFs (183 genes), PbBAMs (17), and Pbrb$H L H$ genes (197) in pears [47-49].

As a large family in plant TFs, $b Z I P$ genes have been found to be involved in several important biological activities [7]. However, the PbrbZIP family has not been studied in much detail in pears, and the PbrbZIP family genes in pears have been rarely studied. In this study, 92 PbrbZIP genes were identified in Chinese white pears and classified into 14 subgroups based on the phylogenetic analysis, gene structure, and protein conserved motif analysis. Subgroup $\mathrm{S}$ had the largest number of PbrbZIPs, followed by subgroups I and A. Subgroup K had the least PbrbZIPs. These results were similar to the case in A. thaliana [26]. Both intron/exon organizations and protein motif patterns were too diverse according to the analysis of gene and protein structure in the $\mathrm{Pbr}$ $b Z I P$ family. Despite the conserved distribution pattern for exons and untranslated regions (UTRs) in subgroups C, D, E, G, I, and S, many other subgroups exhibited diversity in exon number and structure, consistent with the results of protein pattern analysis. The 20 preserved motifs detected by the online MEME program indicated that the subgroup division of the PbrbZIP gene family might have occurred at an earlier stage, and PbrbZIP genes might have played multiple roles in the evolutionary process of adaptation to environmental stresses.

The results of gene duplication analysis showed that WGD/segment events drove the expansion of the $\mathrm{Pbr}$ bZIP gene family. Sixty-five PbrbZIP genes (78.31\%) were categorized into the WGD/segmental type, and 12 genes (14.46\%) belonged to the dispersed type, which might be due to the high ratio of self-incompatibility and the domestication process of pears. WGD/segment and dispersed duplications played an essential role in expanding the pear $b Z I P$ gene family from the aforementioned results. Based on the estimated results of Ks, PbrbZIP genes were duplicated around the time of the most recent WGD event and some from ancient WGD. The Ka/Ks ratios showed that the PbrbZIP genes evolved primarily through purifying selection.

\footnotetext{
(See figure on next page.)

Fig. 6 Analysis of drought tolerance in the PbrbZIP51-silenced Pyrus betulaefolia plants. Phenotype of 1-month-old PbrbZIP51-silenced plants before and after drought treatment for 15 days (a). Electrolyte leakage (EL) (b). Malondialdehyde (MDA) concentrations after drought treatment (c). Chlorophyll fluorescence imaging of silenced plants and control plants (d). The expression of PbrbZIP51 was detected by RT-PCR (e) and qRT-PCR (f) at 3 days after the injection. Chl content of control and pTRV-PbrbZIP51 silencing plants (pTRV-1, pTRV-2) at the end of the drought stress (g), and the phenotype (h) of control and pTRV-PbrbZIP51 silencing plants after drought treatment, and the Fv/Fm ratios (i). Quantitative measurement of $\mathrm{H}_{2} \mathrm{O}_{2}$ levels after drought treatment $(\mathbf{j})$
} 

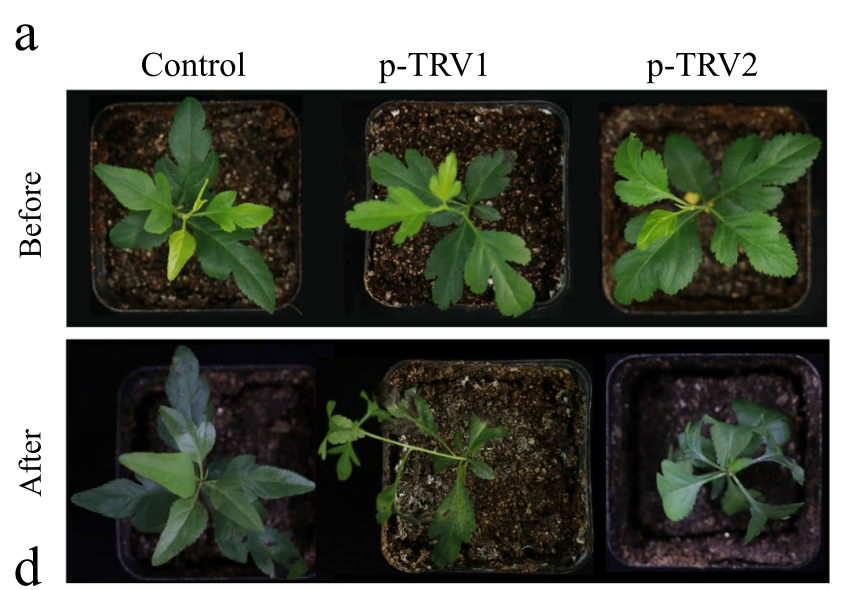

b

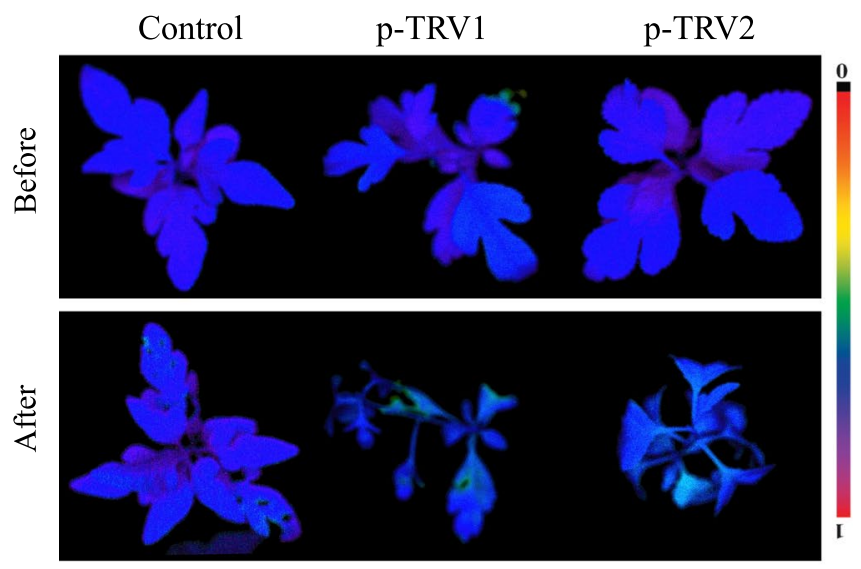

C

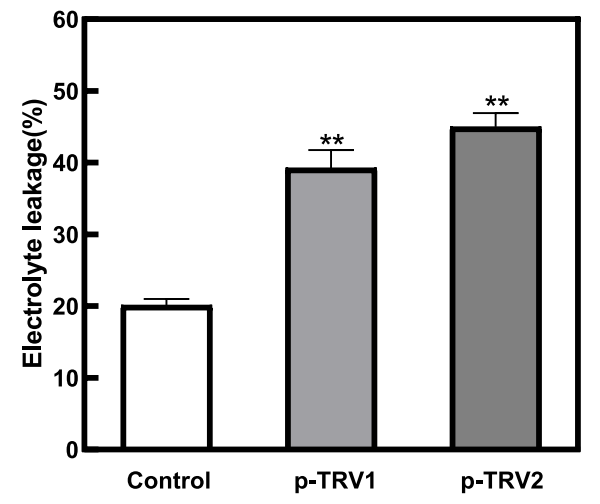

e
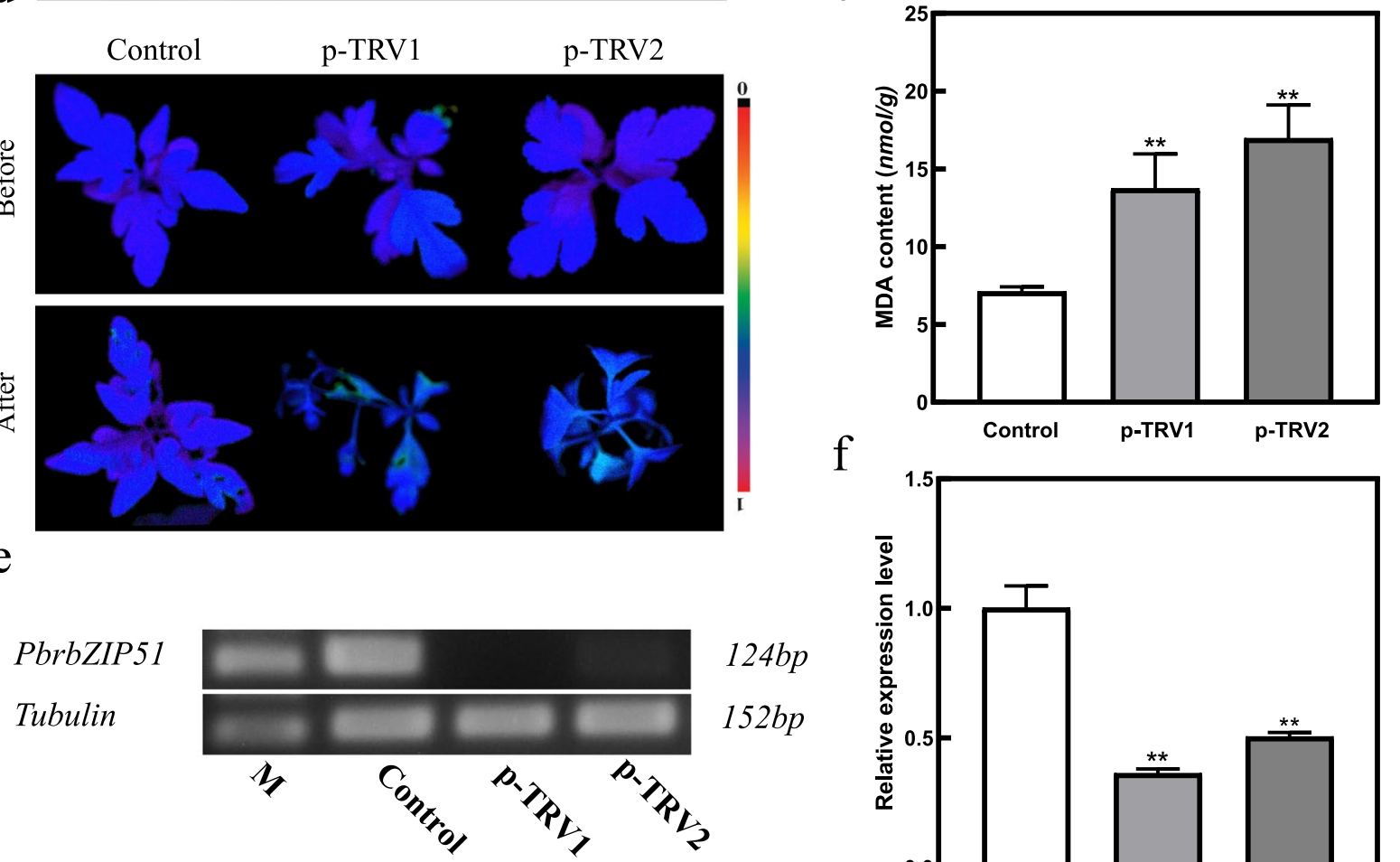

$124 b p$

$152 b p$
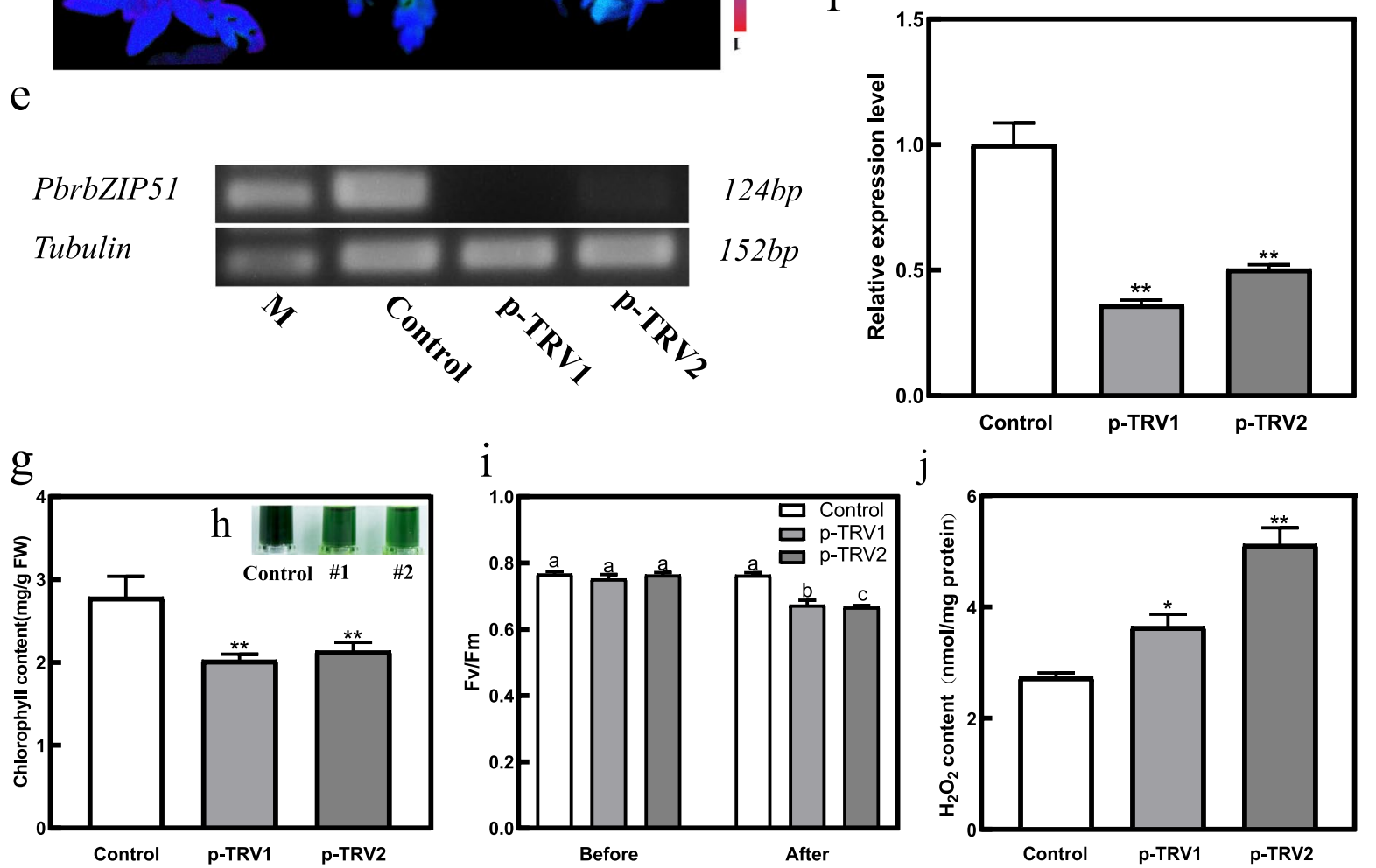

Fig. 6 (See legend on previous page.)

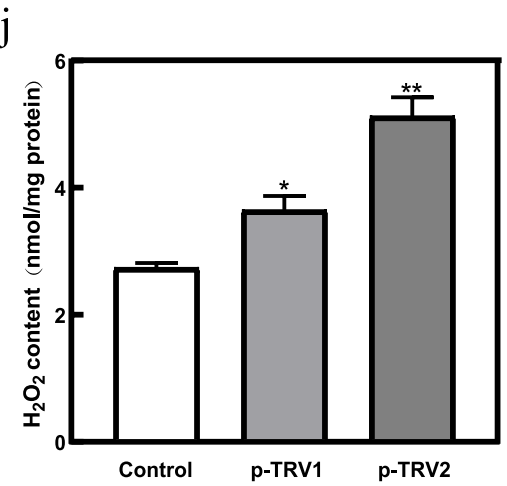


Function enrichment analyses showed that PbrbZIP genes were primarily enriched in functions and processes closely related to TFs, and the pathways they categorized were the principal mechanisms by which bZIP family TFs regulated gene expression downstream, such as hormone signal transduction pathways, circadian rhythm, and MAPK signaling.

Based on the previous transcriptome profile, most $\mathrm{Pbr}$ $b Z I P$ genes were found to be significantly induced by stress treatments. Twenty-three PbrbZIP genes upregulated under cold treatment and 19 differently expressed PbrbZIP genes under drought treatment were detected. In addition, some genes in groups A, C, G, I, and S were possibly involved in biological pathways of drought and cold stress responses. PbrbZIP genes were analyzed using qRT-PCR analysis under stress treatments to verify whether PbrbZIP genes were involved in response to cold or drought stresses. The results showed that the expression of all tested genes was significantly altered under drought or cold treatments. The expression pattern of the same gene between two treatments could be diverse. For instance, under cold treatment, the expression of $\mathrm{Pbr}$ bZIP59 showed an upregulated trend at first before being downregulated. However, under drought stress, it was downregulated first and then upregulated. PbrbZIP16 was more intensively upregulated under cold conditions than under drought stress. In addition, PbrbZIP51, a highly upregulated gene induced under drought stress conditions, has significantly reduced drought tolerance for RNAi pear seedlings. These results indicated that $\mathrm{Pbr}$ $b Z I P$ genes played a role in response to drought and cold stresses in pears, and the processes they were involved in seemed different under various stress conditions. The bZIP TF played an important role in plant regulation and development through protein-protein interactions with variable elements; moreover, the specific functions of genes were realized through the dimer formed by the specific interaction between the monomeric bZIP forms $[50,51]$. However, how the PbrbZIP genes play an important role in the resistance to stress-related injury by regulating the expression level of downstream target genes is still unclear, and the underlying molecular mechanisms require further investigation.

In this study, first the PbrbZIP genes were identified, and subsequently their evolutionary relationship and expression patterns were analyzed under abiotic stresses in pears. PbrbZIPs were involved in the drought and cold stress tolerance pathways by the analyses of qRT-PCR, and the functional analysis indicated that PbrbZIP51 played essential roles in drought stress tolerance in pears. Other genes need to be tested for tolerance to cold and drought stresses in future studies. The results of this study provided a basis for genetic engineering screening of new candidate $b Z I P$ genes in pear cultivars with stress tolerance.

\section{Conclusions}

A total of 92 PbrbZIP genes were identified from the pear genome, which were divided into 14 subgroups based on the results of protein motifs and intron/exon characteristics and phylogenetic analysis. The recent WGD ( 30-45 MYA) and dispersed duplications may be the main driving force for the large-scale amplification of the $b Z I P$ gene family in Chinese white pears. The large-scale amplification of genes in the PbrbZIP family has been proven to be driven by purifying selection. Besides, transcriptome sequencing profile, analyses of qRT-PCR, and VIGS indicated that PbrbZIP genes might play a vital role in response to drought and cold stresses, and the pathway they participated in might differ in response to drought and cold stresses. These results may be useful in developing strategies to increase tolerance further to stress in pears, and providing a foundation for advanced studies to evaluate the mechanisms of $b Z I P$ gene tolerance to cold and drought stresses in pears.

\section{Methods}

\section{Plant materials and bacterial strains}

Pear seeds (Pyrus.betulifolia) were obtained from our pear germplasm orchard of the Center of Pear Engineering Technology Research situated at Hushu in Nanjing. Pear seedlings were grown in a greenhouse with $16 \mathrm{~h} / 8 \mathrm{~h}$ light/dark photoperiod, $75 \%$ relative humidity and $25^{\circ} \mathrm{C}$. Agrobacterium tumefaciens GV3101 was grown in LB media supplemented with kanamycin and Rif at $28^{\circ} \mathrm{C}$ in an orbital shaker at $200 \mathrm{rpm}$ and harvested during the log phase of growth for infiltration.

\section{Identification of bZIP genes}

The whole-genome sequence of Chinese White pears along with GFF3 (general feature format file) was derived from (http://peargenome.njau.edu.cn), and the seed files of bZIP conserved domain (PF00170, PF07716 and PF07777) were downloaded from the Pfam database (http://pfam.sanger.ac.uk/). The conserved Pfam domain was detected by running the Hidden Markov Model (HMM) software, E-value $<0.05$ [52]. Additionally, online SMART program (http://smart.embl-heidelberg.de/) and NCBI Batch CD-search tool were used to detect the existence of the conserved bZIP domain in each protein sequence [53]. The annotation information of the Chinese white pear genome was fetched from the GFF file, and the $\mathrm{R}$ script was used to display the result. The published bZIP protein sequence of $A$. thaliana was downloaded from the TAIR database (http:// www.arabidopsis. org/). 


\section{Sequence and phylogenetic analyses}

We imported the pear and A. thaliana bZIP protein sequences into MEGA 7 software (http://www.megas oftware.net/) [54] and used ClustalW for multiple sequence alignments. The Neighbor-Joining (NJ) phylogenetic tree was constructed by using MEGA 7 software with the bootstrap set to 1000 . P-distance and pairwise deletion which is one of the optional parameters were considered. The annotation and review of the phylogenic trees were completed by iTOL (https://itol.embl.de/) and EvolView (https://www.evolgenius.info/evolview/).

\section{Gene structure and conserved motif analyses}

Conserved motif analysis was performed by online Multiple Expectation Maximization for Motif Elicitation (MEME) [55] (http://meme.ebi.edu.au/meme/ intro. html) with default parameters, and maximum number of motifs parameter set as 20. The intron/exton structures analysis of $92 \mathrm{PbrbZIP}$ genes was found through general feature format (GFF3) files and visualized by using Gene Structure Display Server [56] (http://gsds.cbi.pku.edu. $\mathrm{cn} /)$.

\section{Chromosomal localization and synteny analyses}

The chromosome location information was taken from the GFF file. The synteny among PbrbZIPs was analyzed using the same procedure used in the PGDD (http:// chibba.agtec.uga.edu/duplication/). Primarily, local allvs-all BLASTP research among the identified PbrbZIP genes was carried out $\left(\mathrm{E}<1 \mathrm{e}^{-10}\right)$. Later, MCScanX was used for the determination of syntenic gene pairs with the BLASTP result and gene location information used as input files [57]. Singleton, dispersed, proximal, tandem and WGD/segmental duplications of PbrbZIPs were identified by employing the downstream analysis tool (duplicate_gene_classifier) in the MCScanX package. Results were displayed with the circos-0.69 software [58]. The Ka and Ks values were analyzed using KaKs-calculator 2.0 [59]. The date of segmental duplication events was estimated by using the mean Ks value which considered the succeeding pairs of homologous genes within $100 \mathrm{~Kb}$ on each side of the PbrbZIPs.

\section{Expression analysis of PbrbZIPs under cold and drought stress conditions}

Published transcriptomic data (FPKM values) characterizing the total RNA of drought treatment samples, including D0, D1, D3, D6 (harvested at $0 \mathrm{hpt}, 1 \mathrm{hpt}, 3 \mathrm{hpt}$ and 6 hpt under drought stress) were downloaded from Li et al. (2016) [60]. Cold treatment samples, including C0, C5, C12, C16 (harvested at $0 \mathrm{hpt}, 5 \mathrm{hpt}, 12 \mathrm{hpt}$ and 24 hpt under cold stress) were downloaded from Yang and Huang (2018) [61]. The expression patterns of PbrbZIPs under drought and cold stress were determined, and the differentially expressed genes were identified with the threshold $\left|\log 2^{\mathrm{FC}}\right|>1$. These results were visualized by TBtools v1.082 [62].

For the expression analysis, nine-week-old pear seedlings, exposed to drought and cold stress, were used to test the relative transcript level of selected genes by qRT-PCR. The seedlings were placed in a chamber set at $4{ }^{\circ} \mathrm{C}$ for $0 \mathrm{hpt}, 2 \mathrm{hpt}, 3 \mathrm{hpt}, 6 \mathrm{hpt}, 12 \mathrm{hpt}$ and $24 \mathrm{hpt}$. For drought treatment, the seedlings were placed on dry filter papers for $0 \mathrm{hpt}, 1 \mathrm{hpt}, 3 \mathrm{hpt}, 9 \mathrm{hpt}, 12 \mathrm{hpt}$ and 24 hpt under ambient environment. The total RNA of the pear was extracted as instructed in RNA kit (Tiangen, Beijing, China), and the cDNA was synthesized using PrimeScript RT (Trans Gen) reagent kit. NCBI online tool Primer-BLAST (https://www.ncbi.nlm.nih.gov/tools/ primer-blast/index.cgi? LINK LOC=Blast Home) was used to design the specialized primers of the constitutive TUB (Pbr042345.1, as internal control) [63] and eight tested PbrbZIPs (Table S5). As previously reported, we used SYBR ${ }^{\circledR}$ Green Premix kit (TaKaRa Biotechnology, Dalian, China) to perform qRT-PCR on a Lightcycler 480 (Roche), and the PCR mixture was composed of $10 \mu \mathrm{l} 2$ SYBR PremixExTaq ${ }^{\mathrm{TM}}, 2.5 \mu \mathrm{l}$ per primer and $1 \mu \mathrm{l}$ cDNA model in a final volume of $20 \mu \mathrm{l}$ [48]. $2^{-\Delta \Delta \mathrm{Ct}}$ method was used to evaluate the expression of PbrbZIPs under cold and drought stress conditions.

\section{Generation of silent plants and physiological analyses}

As previously reported, the expression of PbrbZIP51 was suppressed by virus-induced gene silencing (VIGS) -mediated method $[47,64]$. Non-injected leaves of each plant were collected and submitted to genomic PCR and qRT-PCR to analyze whether PbrbZIP51 was silenced after 3 days, and the silenced plants exhibiting similar magnitude of PbrbZIP51 suppression were used for further drought treatment. Electrolyte Leakage was measured by conductivity monitor according to prior method [65]. Chlorophyll was extracted and analyzed in accordance with prior method [66]. $\mathrm{MDA}, \mathrm{H}_{2} \mathrm{O}_{2}$ and $\mathrm{O}_{2}{ }^{-}$content were measured by specific analytical kits (Nanjing Jiancheng Bioengineering Institute, Nanjing, China). The level of the chlorophyll fluorescence was measured by Imaging PAM CHL fluorometer. The detail parameters and the estimate method of $\mathrm{Fv} / \mathrm{Fm}$ values were described by Woo et al. (Walz, Germany) [67].

\section{Statistical analysis}

In this study, abiotic stresses and qRT-PCR expression pattern data were repeated a minimum of three times. The data in the figures were presented in the form of an average \pm standard error (SE). All data was analyzed by $\mathrm{T}$-test function in R-language to test the significance 
level of data between the treatment and the control groups $(* P<0.05, * * P<0.01)$.

\begin{abstract}
Abbreviations
bZIP: Basic region-leucine zipper; TF: Transcription factor; Abscisic Acid: ABA; AsA: Ascorbic acid; ROS: Reactive oxygen species; ABRE: ABA responsive element; PI: Protein isoelectric points; GRAVY: Grand average of hydropathy; SMART: Simple Modular Architecture Research Tool; NCBI: National Center for Biotechnology Information; Ks: Synonymous substitutions per site; Ka: Nonsynonymous substitutions per nonsynonymous site; hpt: Hours post treatment; EL: Electrolyte leakage; MAPK: Mitogen-activated protein kinase; MDA: Malondialdehyde; HMM: Hidden Markov Model; GSDS: Gene Structure Display Server; MEME: Multiple Expectation Maximization for Motif Elicitation; NJ: Neighbor-Joining; UTR: Untranslated regions; WGD: Whole-genome duplications; MYA: Millions of years Ago; qRT-PCR: Quantitative real-time polymerase chain reaction; VIGS: Virus-induced gene silencing; ORF: Open reading frame; MES: 2-(Nmorpholino) ethanesulfonic acid.
\end{abstract}

\section{Supplementary Information}

The online version contains supplementary material available at https://doi. org/10.1186/s12870-021-03356-0.

Additional file 1 : Figure S1. Phylogenetic tree of 78 AtbZIPs and the 92 PbrbZIPs proteins. The phylogenetic tree based on the protein sequences was built by MEGA 7. The annotation and review of the phylogenic tree was completed by Evolview (https://www.evolgenius.info/evolview/).

Additional file 2 : Figure S2. Functional annotation enrichment analysis. Term enrichment analysis of PbrbZIP proteins. (b) KEGG enrichment analysis of PbrbZIP proteins.2.

Additional file 3 : Figure S3. Molecular identification of Pbrbzip51silenced pear and other original images of Fig.6. Semi-quantitative RT-PCR analysis identification of the plants used specific primers of PbrbZIP51. M DNA marker (DL 2000); Control, untransformed plants. Numbers on the top of the gel panels indicate the PbrbZIP51-silenced lines.

Additional file 4 : Table S1. Detailed characteristics of PbrbZIPs.

Additional file $\mathbf{5}$ : Table S2. Sequence information of 20 detected motifs in MEME analysis.

Additional file 6 : Table S3. Duplication type of PbrbZIP genes in pear (Pyrus bretschneideri).

Additional file 7 : Table S4. Differentially expressed genes after stress treatments.

Additional file 8 : Table S5. Primers of PbrbZIPs for qRT-PCR and vector construction.

\section{Acknowledgements}

Bioinformatic analysis was supported by the Bioinformatics Center of Nanjing Agricultural University.

\section{Authors' contributions}

$\mathrm{XSH}, \mathrm{MM}$ and HZD designed and carried out the experiments, QMC and MM performed all bioinformatic analyses and wrote the manuscript. XSH and SLZ directed and revised the manuscript. All authors read, reviewed and approved the final manuscript.

\section{Funding}

This work has been supported by the National Key Research and Development Program of China (2019YFD1000102), the National Science Foundation of China (31872070; 32072538), the Jiangsu Agriculture Science and Technology Innovation Fund (CX(18)3065), the Excellent Youth Natural Science Foundation of Jiangsu Province (SBK2017030026), the Fundamental Research Funds for the Central Universities of Nanjing Agricultural University (KYZ201607), the SRT project of the Nanjing Agriculture University (202011YX05).

\section{Availability of data and materials}

All needed genome sequences and genome annotation files of Chinese white pear were obtained from the Nanjing Agricultural University pear genome project website (http://peargenome.njau.edu.cn), and the published bZIP sequences of $A$. thaliana were acquired from the TAIR database (http://www. arabidopsis.org/). The cold transcriptome sequencing data used in this study was got from the additional files from previous report (https://doi.org/10. 1016/j.gene.2018.03.067). The drought transcriptome sequencing raw data used in this study has been uploaded to the NCBI (https://www.ncbi.nlm.nih. gov/Traces/study/?acc $=$ SRP148620). All databases in this study are available to the public.

\section{Declarations}

\section{Ethics approval and consent to participate}

The genome database of Chinese white pear (Pyrus bretschneideri) is applied for our research under the permission of Center of Pear Engineering Technology Research. The test materials 'Pyrus betulaefolia' were collected from the pear germplasm orchard of the Center of Pear Engineering Technology Research situated at Hushu in Nanjing under the permission of Center of Pear Engineering Technology Research.

\section{Consent for publication}

Not Applicable.

\section{Competing interests}

The authors declare that they have no competing interests.

Received: 21 May 2021 Accepted: 23 November 2021

Published online: 09 December 2021

\section{References}

1. Pérez-Rodríquez P, Riaño-Pachón DM, Corrêa LG, Rensing SA, Kersten B, Mueller-Roeber B. PInTFDB: updated content and new features of the plant transcription factor database. Nucleic Acids Res. 2010;38:D822-7. https://doi.org/10.1093/nar/gkp805.

2. Nijhawan A, Jain M, Tyagi AK, Khurana JP. Genomic survey and gene expression analysis of the basic leucine zipper transcription factor family in rice. Plant Physiol. 2008;146(2):333-50. https://doi.org/10.1104/pp.107. 112821.

3. Jakoby M, Weisshaar B, Dröge-Laser W, Vicente-Carbajosa J, Tiedemann J, Kroj T, et al. bZIP transcription factors in Arabidopsis. Trends Plant Sci. 2002;7(3):106-11. https://doi.org/10.1016/s1360-1385(01)02223-3.

4. Lee SC, Choi HW, Hwang IS, Choi DS, Hwang BK. Functional roles of the pepper pathogen-induced bZIP transcription factor, CAbZIP1, in enhanced resistance to pathogen infection and environmental stresses. Planta. 2006;224(5):1209-25. https://doi.org/10.1007/s00425-006-0302-4.

5. Fukazawa J, Sakai T, Ishida S, Yamaguchi I, Kamiya Y, Takahashi Y. Repression of shoot growth, a bZIP transcriptional activator, regulates cell elongation by controlling the level of gibberellins. Plant Cell. 2000;12(6):90115. https://doi.org/10.1105/tpc.12.6.901.

6. Yin Y, Zhu Q, Dai S, Lamb C, Beachy RN. RF2a, a bZIP transcriptional activator of the phloem-specific rice tungro bacilliform virus promoter, functions in vascular development. EMBO J. 1997;16(17):5247-59. https:// doi.org/10.1093/emboj/16.17.5247.

7. Abe M, Kobayashi Y, Yamamoto S, Daimon Y, Yamaguchi A, Ikeda Y, et al. $\mathrm{FD}$, a bZIP protein mediating signals from the floral pathway integrator FT at the shoot apex. Science. 2005;309(5737):1052-6. https://doi.org/10. 1126/science.1115983.

8. Shen H, Cao K, Wang X. A conserved proline residue in the leucine zipper region of AtbZIP34 and AtbZIP61 in Arabidopsis thaliana interferes with the formation of homodimer. Biochem Biophys Res Commun. 2007;362(2):425-30. https://doi.org/10.1016/j.bbrc.2007.08.026.

9. Baena-González E, Rolland F, Thevelein JM, Sheen J. A central integrator of transcription networks in plant stress and energy signalling. Nature. 2007:448(7156):938-42. https://doi.org/10.1038/nature06069.

10. Lara P, Onate-Sanchez L, Abraham Z, Ferrandiz C, Diaz I, Carbonero P, et al. Synergistic activation of seed storage protein gene expression in 
Arabidopsis by ABI3 and two bZIPs related to OPAQUE2. J Biol Chem. 2003;278(23):21003-11. https://doi.org/1 0.1074/jbc.M210538200.

11. Nieva C, Busk PK, Domínguez-Puigjaner E, Lumbreras V, Testillano PS, Risueño MC, et al. Isolation and functional characterisation of two new bZIP maize regulators of the ABA responsive gene rab28. Plant Mol Biol. 2005;58(6):899-914. https://doi.org/10.1007/s1 1103-005-8407-x.

12. Uno Y, Furihata T, Abe H, Yoshida R, Shinozaki K, Yamaguchi-Shinozaki K. Arabidopsis basic leucine zipper transcription factors involved in an abscisic acid-dependent signal transduction pathway under drought and high-salinity conditions. Proc Natl Acad Sci U S A. 2000;97(21):11632-7. https://doi.org/10.1073/pnas.190309197.

13. Ulm R, Baumann A, Oravecz A, Máté Z, Adám E, Oakeley EJ, et al. Genome-wide analysis of gene expression reveals function of the bZIP transcription factor HY5 in the UV-B response of Arabidopsis. Proc Natl Acad Sci U S A. 2004;101(5):1397-402. https://doi.org/10.1073/pnas. 0308044100.

14. Wellmer F, Kircher S, Rügner A, Frohnmeyer H, Schäfer E, Harter K. Phosphorylation of the parsley bZIP transcription factor CPRF2 is regulated by light. J Biol Chem. 1999;274(41):29476-82. https://doi.org/10.1074/jbc. 274.41.29476.

15. Kaminaka H, Näke C, Epple P, Dittgen J, Schütze K, Chaban C, et al. bZIP10-LSD1 antagonism modulates basal defense and cell death in Arabidopsis following infection. EMBO J. 2006;25(18):4400-11. https://doi. org/10.1038/sj.emboj.7601312.

16. Thurow C, Schiermeyer A, Krawczyk S, Butterbrodt T, Nickolov K, Gatz C. Tobacco bZIP transcription factor TGA2.2 and related factor TGA2.1 have distinct roles in plant defense responses and plant development. Plant J. 2005;44(1):100-13. https://doi.org/10.1111/j.1365-313X.2005.02513.X.

17. Liu C, Mao B, Ou S, Wang W, Liu L, Wu Y, et al. OsbZIP71, a bZIP transcription factor, confers salinity and drought tolerance in rice. Plant Mol Biol. 2014:84(1-2):19-36. https://doi.org/10.1007/s11103-013-0115-3.

18. Ying S, Zhang DF, Fu J, Shi YS, Song YC, Wang TY, et al. Cloning and characterization of a maize bZIP transcription factor, ZmbZIP72, confers drought and salt tolerance in transgenic Arabidopsis. Planta. 2012;235(2):253-66. https://doi.org/10.1007/s00425-011-1496-7.

19. Chinnusamy V, Ohta M, Kanrar S, Lee BH, Hong X, Agarwal M, et al. ICE1: a regulator of cold-induced transcriptome and freezing tolerance in Arabidopsis. Genes Dev. 2003;17(8):1043-54. https://doi.org/10.1101/gad. 1077503.

20. Kiribuchi K, Sugimori M, Takeda M, Otani T, Okada K, Onodera H, et al. RERJ1, a jasmonic acid-responsive gene from rice, encodes a basic helixloop-helix protein. Biochem Biophys Res Commun. 2004;325(3):857-63. https://doi.org/10.1016/j.bbrc.2004.10.126.

21. Liao Y, Zou HF, Wei W, Hao YJ, Tian AG, Huang J, et al. Soybean GmbZIP44, GmbZIP62 and GmbZIP78 genes function as negative regulator of ABA signaling and confer salt and freezing tolerance in transgenic Arabidopsis. Planta. 2008;228(2):225-40. https://doi.org/10.1007/ s00425-008-0731-3.

22. Yang $\mathrm{S}, \mathrm{Xu} \mathrm{K}$, Chen $\mathrm{S}, \mathrm{Li}$, Xia H, Chen L, et al. A stress-responsive bZIP transcription factor OsbZIP62 improves drought and oxidative tolerance in rice. BMC Plant Biol. 2019;19(1):260. https://doi.org/10.1186/ s12870-019-1872-1.

23. Ma H, Liu C, Li Z, Ran Q, Xie G, Wang B, et al. ZmbZIP4 contributes to stress resistance in maize by regulating $A B A$ synthesis and root development. Plant Physiol. 2018;178(2):753-70. https://doi.org/10.1104/pp.18. 00436.

24. Tu $M$, Wang $X$, Feng $T$, Sun $X$, Wang $Y$, Huang $L$, et al. Expression of a grape (Vitis vinifera) bZIP transcription factor, VIbZIP36, in Arabidopsis thaliana confers tolerance of drought stress during seed germination and seedling establishment. Plant Sci. 2016;252:311-23. https://doi.org/10.1016/j. plantsci.2016.08.011.

25. An JP, Yao JF, Wang XN, You CX, Wang XF, Hao YJ. MdHY5 positively regulates cold tolerance via CBF-dependent and CBF-independent pathways in apple. J Plant Physiol. 2017;218:275-81. https://doi.org/10.1016/j.jplph. 2017.09.001.

26. Droge-Laser W, Snoek BL, Snel B, Weiste C. The Arabidopsis bZIP transcription factor family-an update. Curr Opin Plant Biol. 2018;45(Pt A):36-49. https://doi.org/10.1016/.jpbi.2018.05.001.

27. Wei K, Chen J, Wang Y, Chen Y, Chen S, Lin Y, et al. Genome-wide analysis of bzIP-encoding genes in maize. DNA Res. 2012;19(6):463-76. https:// doi.org/10.1093/dnares/dss026.
28. Liu J, Chen N, Chen F, Cai B, Dal Santo S, Tornielli GB, et al. Genome-wide analysis and expression profile of the bZIP transcription factor gene family in grapevine (Vitis vinifera). BMC Genomics. 2014;15:281. https://doi. org/10.1186/1471-2164-15-281.

29. Zhao J, Guo R, Guo C, Hou H, Wang X, Gao H. Evolutionary and expression analyses of the apple basic leucine zipper transcription factor family. Front Plant Sci. 2016;7:376. https://doi.org/10.3389/fpls.2016.00376.

30. Zhao L, Yang T, Xing C, Dong H, Qi K, Gao J, et al. The $\beta$-amylase PbrBAM3 from pear (Pyrus betulaefolia) regulates soluble sugar accumulation and ROS homeostasis in response to cold stress. Plant Sci. 2019;287:110184. https://doi.org/10.1016/j.plantsci.2019.110184.

31. Liu Y, Yang T, Lin Z, Gu B, Xing C, Zhao L, et al. A WRKY transcription factor PbrWRKY53 from Pyrus betulaefolia is involved in drought tolerance and AsA accumulation. Plant Biotechnol J. 2019;17(9):1770-87. https://doi. org/10.1111/pbi.13099.

32. Jha UC, Bohra A, Jha R. Breeding approaches and genomics technologies to increase crop yield under low-temperature stress. Plant Cell Rep. 2017;36(1):1-35. https://doi.org/10.1007/s00299-016-2073-0.

33. Wu J, Wang Z, Shi Z, Zhang S, Ming R, Zhu S, et al. The genome of the pear (Pyrus bretschneideri Rehd.). Genome Res. 2013;23(2):396-408. https://doi.org/10.1101/gr.144311.112.

34. Gangappa SN, Botto JF. The multifaceted roles of HY5 in plant growth and development. Mol Plant. 2016;9(10):1353-65. https://doi.org/10.1016/j. molp.2016.07.002.

35. Zhou F, Sun TH, Zhao L, Pan XW, Lu S. The bZIP transcription factor HY5 interacts with the promoter of the monoterpene synthase gene QH6 in modulating its rhythmic expression. Front Plant Sci. 2015;6:304. https:// doi.org/10.3389/fpls.2015.00304.

36. El Sarraf N, Gurel F, Tufan F, McGuffin L. Characterisation of HvVIP1 and expression profile analysis of stress response regulators in barley under Agrobacterium and fusarium infections. PLoS One. 2019;14(6):e0218120. https://doi.org/10.1371/journal.pone.0218120.

37. Li X, Duan X, Jiang H, Sun Y, Tang Y, Yuan Z, et al. Genome-wide analysis of basic/helix-loop-helix transcription factor family in rice and Arabidopsis. Plant Physiol. 2006;141(4):1167-84. https://doi.org/10.1104/pp.106. 080580.

38. Maher C, Stein L, Ware D. Evolution of Arabidopsis microRNA families through duplication events. Genome Res. 2006;16(4):510-9. https://doi. org/10.1101/gr.4680506.

39. Qiao X, Li M, Li L, Yin H, Wu J, Zhang S. Genome-wide identification and comparative analysis of the heat shock transcription factor family in Chinese white pear (Pyrus bretschneideri) and five other Rosaceae species. BMC Plant Biol. 2015;15:12. https://doi.org/10.1186/s12870-014-0401-5.

40. Fawcett JA, Maere S, Van de Peer Y. Plants with double genomes might have had a better chance to survive the cretaceous-tertiary extinction event. Proc Natl Acad Sci U S A. 2009;106(14):5737-42. https://doi.org/10. 1073/pnas.0900906106.

41. Krishnamurthy P, Hong JK, Kim JA, Jeong MJ, Lee YH, Lee SI. Genomewide analysis of the expansin gene superfamily reveals Brassica rapa-specific evolutionary dynamics upon whole genome triplication. Mol Gen Genomics. 2015;290(2):521-30. https://doi.org/10.1007/ s00438-014-0935-0.

42. Sharma R, Singh G, Bhattacharya S, Singh A. Comparative transcriptome meta-analysis of Arabidopsis thaliana under drought and cold stress. PLoS One. 2018;13(9):e0203266. https://doi.org/10.1371/journal.pone. 0203266.

43. Yang Y, Li J, Li H, Yang Y, Guang Y, Zhou Y. The bZIP gene family in watermelon: genome-wide identification and expression analysis under cold stress and root-knot nematode infection. PeerJ. 2019;7:e7878. https://doi. org/10.7717/peerj.7878.

44. Vigeolas H, Chinoy C, Zuther E, Blessington B, Geigenberger P, Domoney C. Combined metabolomic and genetic approaches reveal a link between the polyamine pathway and albumin 2 in developing pea seeds. Plant Physiol. 2008;146(1):74-82. https://doi.org/10.1104/pp.107. 111369.

45. Golldack D, Lüking I, Yang O. Plant tolerance to drought and salinity: stress regulating transcription factors and their functional significance in the cellular transcriptional network. Plant Cell Rep. 2011;30(8):1383-91. https://doi.org/10.1007/s00299-011-1068-0.

46. Huang XS, Wang W, Zhang Q, Liu JH. A basic helix-loop-helix transcription factor, PtrbHLH, of Poncirus trifoliata confers cold tolerance and 
modulates peroxidase-mediated scavenging of hydrogen peroxide. Plant Physiol. 2013;162(2):1178-94. https://doi.org/10.1104/pp.112.210740.

47. Dong H, Chen Q, Dai Y, Hu W, Zhang S, Huang X. Genome-wide identification of PbrbHLH family genes, and expression analysis in response to drought and cold stresses in pear (Pyrus bretschneideri). BMC Plant Biol. 2021;21(1):86. https://doi.org/10.1186/s12870-021-02862-5.

48. Gong X, Zhao L, Song X, Lin Z, Gu B, Yan J, et al. Genome-wide analyses and expression patterns under abiotic stress of NAC transcription factors in white pear (Pyrus bretschneideri). BMC Plant Biol. 2019;19(1):161 . https://doi.org/10.1186/s12870-019-1760-8.

49. Zhao L, Gong X, Gao J, Dong H, Zhang S, Tao S, et al. Transcriptomic and evolutionary analyses of white pear (Pyrus bretschneideri) $\beta$-amylase genes reveals their importance for cold and drought stress responses. Gene. 2019;689:102-13. https://doi.org/10.1016/j.gene.2018.11.092.

50. Ehlert A, Weltmeier F, Wang X, Mayer CS, Smeekens S, Vicente-Carbajosa J, et al. Two-hybrid protein-protein interaction analysis in Arabidopsis protoplasts: establishment of a heterodimerization map of group $C$ and group S bZIP transcription factors. Plant J. 2006;46(5):890-900. https://doi. org/10.1111/j.1365-313X.2006.02731.X.

51. Sornaraj P, Luang S, Lopato S, Hrmova M. Basic leucine zipper (bZIP) transcription factors involved in abiotic stresses: a molecular model of a wheat bZIP factor and implications of its structure in function. Biochim Biophys Acta. 2016;1860(1 Pt A):46-56. https://doi.org/10.1016/j.bbagen. 2015.10.014.

52. Eddy SR. Accelerated profile HMM searches. PLoS Comput Biol. 2011;7(10):e1002195. https://doi.org/10.1371/journal.pcbi.1002195.

53. Letunic I, Doerks T, Bork P. SMART 7: recent updates to the protein domain annotation resource. Nucleic Acids Res. 2012;40:D302-5. https://doi.org/ 10.1093/nar/gkr931.

54. Kumar S, Stecher G, Li M, Knyaz C, Tamura K. MEGA X: molecular evolutionary genetics analysis across computing platforms. Mol Biol Evol. 2018;35(6):1547-9. https://doi.org/10.1093/molbev/msy096.

55. Bailey TL, Williams N, Misleh C, Li WW. MEME: discovering and analyzing DNA and protein sequence motifs. Nucleic Acids Res. 2006;34:W369-73. https://doi.org/10.1093/nar/gkl198.

56. Hu B, Jin J, Guo AY, Zhang H, Luo J, Gao G. GSDS 2.0: an upgraded gene feature visualization server. Bioinformatics. 2015;31 (8):1296-7. https://doi. org/10.1093/bioinformatics/btu817.

57. Tang H, Wang X, Bowers JE, Ming R, Alam M, Paterson AH. Unraveling ancient hexaploidy through multiply-aligned angiosperm gene maps. Genome Res. 2008;18(12):1944-19454. https://doi.org/10.1101/gr.080978. 108.

58. Krzywinski M, Schein J, Birol I, Connors J, Gascoyne R, Horsman D, et al. Circos: an information aesthetic for comparative genomics. Genome Res. 2009;19(9):1639-45. https://doi.org/10.1101/gr.092759.109.

59. Wang D, Zhang Y, Zhang Z, Zhu J, Yu J. KaKs_Calculator 2.0: a toolkit incorporating gamma-series methods and sliding window strategies. Genomics Proteomics Bioinformatics. 2010;8(1):77-80. https://doi.org/10. 1016/s1672-0229(10)60008-3.

60. Li KQ, Xu XY, Huang XS. Identification of differentially expressed genes related to dehydration resistance in a highly drought-tolerant pear, Pyrus betulaefolia, as through RNA-Seq. PLoS One. 2016;11(2):e0149352. https://doi.org/10.1371/journal.pone.0149352.

61. Yang T, Huang XS. Deep sequencing-based characterization of transcriptome of Pyrus ussuriensis in response to cold stress. Gene. 2018:661:10918. https://doi.org/10.1016/j.gene.2018.03.067.

62. Chen C, Chen H, Zhang Y, Thomas HR, Frank MH, He Y, et al. TBtools: An integrative toolkit developed for interactive analyses of big biological data. Mol Plant. 2020;13(8):1194-202. https://doi.org/10.1016/j.molp. 2020.06.009.

63. Livak KJ, Schmittgen TD. Analysis of relative gene expression data using real-time quantitative PCR and the 2(-Delta Delta $C(T)$ ) method. Methods. 2001;25(4):402-8. https://doi.org/10.1006/meth.2001.1262.

64. Jiang X, Zhang C, Lü P, Jiang G, Liu X, Dai F, et al. RhNAC3, a stressassociated NAC transcription factor, has a role in dehydration tolerance through regulating osmotic stress-related genes in rose petals. Plant Biotechnol J. 2014;12(1):38-48. https://doi.org/10.1111/pbi.12114.

65. Dahro B, Wang F, Peng T, Liu JH. PtrA/NINV, an alkaline/neutral invertase gene of Poncirus trifoliata, confers enhanced tolerance to multiple abiotic stresses by modulating ROS levels and maintaining photosynthetic efficiency. BMC Plant Biol. 2016;16:76. https://doi.org/10.1186/ s12870-016-0761-0.

66. Liu JH, Moriguchi T. Changes in free polyamine titers and expression of polyamine biosynthetic genes during growth of peach in vitro callus. Plant Cell Rep. 2007;26(2):125-31. https://doi.org/10.1007/ s00299-006-0223-5.

67. Woo NS, Badger MR, Pogson BJ. A rapid, non-invasive procedure for quantitative assessment of drought survival using chlorophyll fluorescence. Plant Methods. 2008;4:27. https://doi.org/10.1186/1746-4811-4-27.

\section{Publisher's Note}

Springer Nature remains neutral with regard to jurisdictional claims in published maps and institutional affiliations.
Ready to submit your research? Choose BMC and benefit from:

- fast, convenient online submission

- thorough peer review by experienced researchers in your field

- rapid publication on acceptance

- support for research data, including large and complex data types

- gold Open Access which fosters wider collaboration and increased citations

- maximum visibility for your research: over $100 \mathrm{M}$ website views per year

At BMC, research is always in progress.

Learn more biomedcentral.com/submissions 